\title{
From Personalized Medicine to Population Health: A Survey of mHealth Sensing Techniques
}

\author{
Zhiyuan Wang, Haoyi Xiong, Jie Zhang, Sijia Yang, Mehdi Boukhechba, \\ Laura E. Barnes, and Daqing Zhang, Fellow, IEEE
}

\begin{abstract}
Mobile Sensing Apps have been widely used as a practical approach to collect behavioral and health-related information from individuals and provide timely intervention to promote health and well-beings, such as mental health and chronic cares. As the objectives of mobile sensing could be either (a) personalized medicine for individuals or (b) public health for populations, in this work we review the design of these mobile sensing apps, and propose to categorize the design of these apps/systems in two paradigms - (i) Personal Sensing and (ii) Crowd Sensing paradigms. While both sensing paradigms might incorporate with common ubiquitous sensing technologies, such as wearable sensors, mobility monitoring, mobile data offloading, and/or cloudbased data analytics to collect and process sensing data from individuals, we present a novel taxonomy system with two major components that can specify and classify apps/systems from aspects of the life-cycle of mHealth Sensing: (1) Sensing Task Creation \& Participation, (2) Health Surveillance \& Data Collection, and (3) Data Analysis \& Knowledge Discovery. With respect to different goals of the two paradigms, this work systematically reviews this field, and summarizes the design of typical apps/systems in the view of the configurations and interactions between these two components. In addition to summarization, the proposed taxonomy system also helps figure out the potential directions of mobile sensing for health from both personalized medicines and population health perspectives.
\end{abstract}

Index Terms-Mobile Health (mHealth), Mobile Sensing, Mobile Crowd Sensing (MCS), and Personal Sensing.

\section{INTRODUCTION}

Mobile Sensing [1] refers to a sensing paradigm leveraging the sensors embedded in the ubiquitous mobile devices, such as mobile phones, smartwatches, and other wearable devices. Lots of work studied the adoption of mobile sensing techniques in health domains [2], [3] such as mental health [4] and chronic cares [5]. Early visionary works [6], [7] proposed the basic framework of mobile health (mHealth) sensing techniques in nowadays that leverage "non-invasive" mobile sensing schemes [8] to collect data for human activities recognition and infer the individual's health status using machine learning algorithms with longitude and real-time sensory data accordingly [4], [9]-[11]. Though there are several works reviewing and surveying the research problems [12]-[16], emerg-

Z. Wang and H. Xiong are with the Big Data Lab, Baidu Research, Baidu Inc., Haidian, Beijing, China.

Z. Wang, M. Boukhechba, and L.E Barnes are with the Department of Engineering Systems and Environment, University of Virginia, Charlottesville, Virginia, United States.

J. Zhang and D. Zhang are with the Department of Computer Science, Peking University, Haidian, Beijing, China.

S. Yang is with the School of Cyberspace Security, Beijing University of Posts and Telecommunications, Haidian, Beijing, China.

Corresponding Author: Haoyi Xiong, haoyi.xiong.fr@ieee.org ing techniques [17]-[19], system design [20]-[22], and prototyping tools [11], [23], [24] for mobile sensing for health (mHealth Sensing) apps/systems, to the best of our knowledge, the motivations of mHealth Sensing, in addition to tracking individuals' behaviors related to some specific health issues, are not well categorized with respects to two perspectives of modern healthcare as follows.

- Personalized Medicine. The personalized medicine focuses on individual patients-"with medical decisions, practices, interventions and/or products being tailored to the individual patient based on their predicted response or risk of disease" [25]. Thus, the objective of personalized medicine is to improve and optimize the individual treatment effects through sensing, monitoring, and predicting their health status [26], [27].

- Population Health. The population health is defined as "the health outcomes [28] of a group of individuals, including the distribution of such outcomes within the group" [29]. The goal of population health is to promote the health of an entire human population [30], where the approaches include discovering health outcomes, understanding patterns of health determinants, and policy making for interventions.

With two types of healthcare outcomes expected [31]-[33], it is reasonable to assume the way that existing works designed, implemented mHealth Sensing apps and kept participants engaged might be significantly different. Thus, we propose to provide a systematic review on mHealth Sensing with details in taxonomies from the perspectives of sensing objectives to motivate the creation of a mHealth app, and sensing paradigms for mHealth app designs and implementations (D\&Is), namely sensing paradigms and $D \& I s$.

Sensing Objectives. In this paper, we propose to specify the sensing objectives of mHealth apps as (a) personalized medicine for individuals (shortly Personalized Medicine) and (b) public health for populations (shortly Population Health) which mainly aim to monitor personalized or population health dynamics and gaining health-related knowledge on individuals or public health issues, respectively. For example, some of mHealth apps for Sports [34], [35] monitor individual runners' physical indicators in a real-time manner, detect the movements when body functioning abnormally, and provide just-intime intervention, while some other works propose to deploy mHealth apps among a groups of athletes, and collect and further analyze sport-related data to understand their training habits and efficiency [36], [37]. 


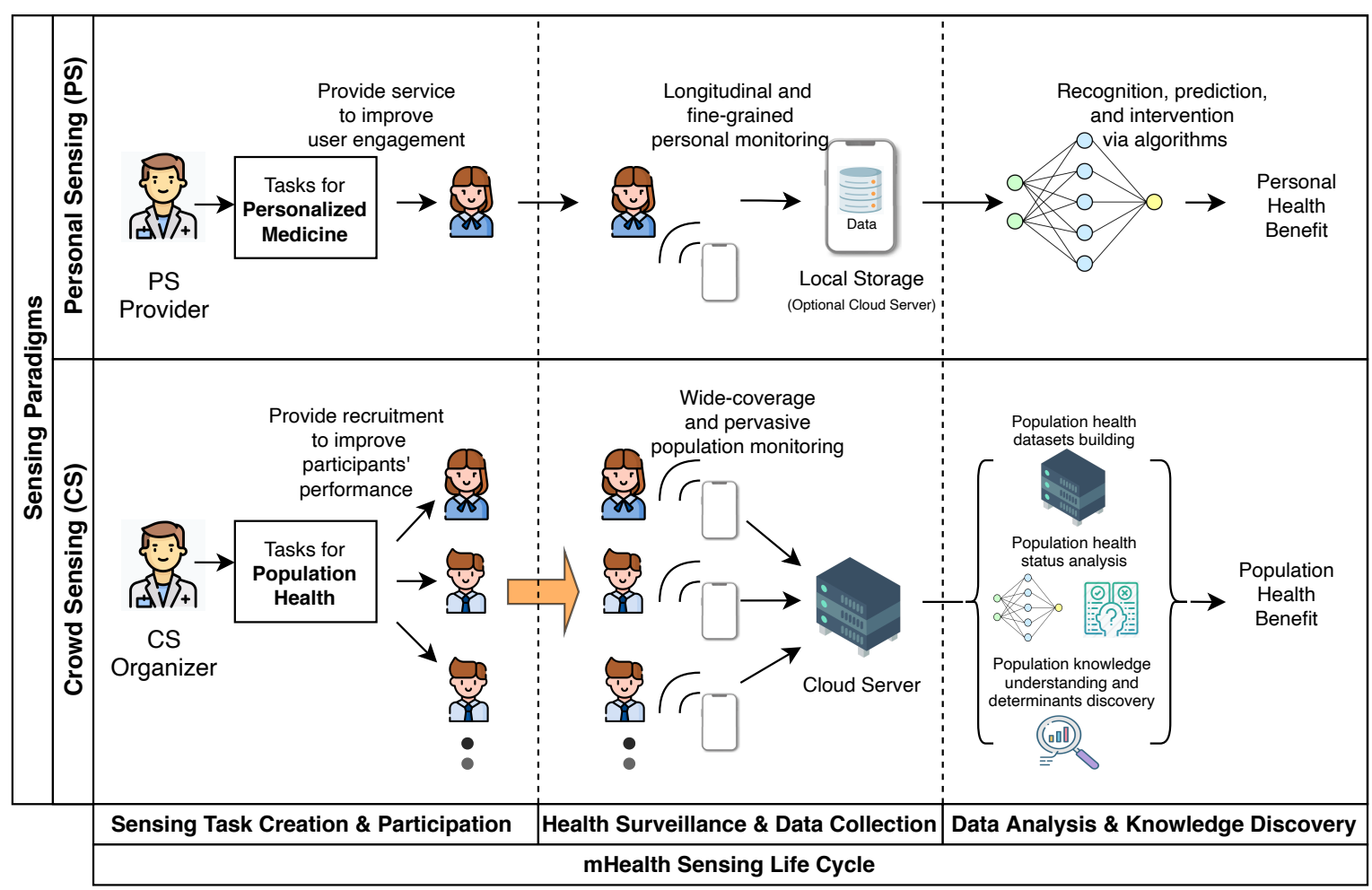

Fig. 1: The three-stage life cycle of the mHealth Sensing apps from Personal Sensing for Personalized Medicine and Crowd Sensing for Population Health perspectives

Sensing Paradigms and D\&Is. For the two perspectives of sensing objectives, we plan to generalize and categorize mHealth apps into two sensing paradigms - (i) Personal Sensing and (ii) Crowd Sensing paradigms, according to the significant differences in app designs and implementations such as user engagement strategies [38], [39] and data analysis approaches [40], [41]. Specifically, Personal Sensing paradigm aims at specific personalized health modeling, which probes and profiles digital biomarkers and vibrations of individual users; Crowd Sensing paradigm is to achieve comprehensive population health analysis, which surveys and understands the patterns and knowledge of public health problems among populations.

To this end, we intend to establish novel taxonomy systems to specify and classify mHealth Sensing apps/systems respect to unique sensing objectives and paradigms. To further divide and analyze each component of mHealth apps/systems in their life cycle, inspired by the four-fold life cycle of Mobile Crowd Sensing proposed by Zhang et al. [42], we generalize the lifecycle of mHealth Sensing for both Sensing Objectives and Sensing Paradigms and D\&Is into three steps:

1) Sensing Task Creation \& Participation - With a pool of potential mobile users, the mHealth Sensing organizers create tasks for specific health issues via deployed apps [2], [43], [44], then prompt the participation of the users [7], [45], [46] or recruitment participants with incentives [47], [48].

2) Health Surveillance \& Data Collection - With actively engaged participants, the mHealth Sensing apps and systems collect health-related data from participants in their daily life scenarios [8], [49], then store and offload the sensing data accordingly [50], [51].

3) Data Analysis \& Knowledge Discovery - With healthrelated data collected, the mHealth Sensing apps and systems carry out data processing and analysis to predict health-related events for individuals [52], [53] and discover determinants of health [29] - i.e., knowledge about population health and well-beings [7], [54].

With proposed taxonomy systems and life cycle of mHealth Sensing apps, this work systematically reviews mHealth Sensing fields, and summarizes several typical apps in the view of the configurations and interactions between these two type of apps. On the basis of the above, we intend to make the following contributions:

- Reviewing and distinguishing existing mHealth Sensing apps in the objectives of Personalized Medicine and Population Health, and categorizing them into Personal Sensing and Crowd Sensing paradigms respectively with the applications on two common health issues as case studies.

- Proposing two novel taxonomy systems to specify and classify the two kinds of mHealth Sensing apps by sensing objectives and sensing paradigms components, and generalizing the life cycle of mHealth Sensing paradigms as (1) Sensing Task Creation \& Participation, (2) Health Surveillance \& Data Collection, and (3) Data Analysis \& Knowledge Discovery to characterize the taxonomy systems and research problems in a structured way.

- Identifying the research gaps in future researches on mHealth Sensing. By systematically studying and classifying the existing mHealth apps leveraging our proposed 
taxonomy systems and three-fold life-cycle framework, we thoroughly summarize and distinguish the main research directions in health-related fields and foresee the potential problems for mHealth researches by applying the methodology proposed in this survey.

The rest of this manuscript is organized as follows. Section II reviews typical mHealth Sensing apps and systems for common health issues, where we discuss three common issues as case studies in details. Section III introduces mHealth Sensing apps' taxonomy system from the sensing objectives perspective. Section IV presents the taxonomy system of mHealth apps D\&I issues from the perspective of sensing paradigms. In section V, we point out identified research gaps and future directions in mHealth Sensing field, while section VI concludes the article.

\section{Reviewing mHealth Sensing Apps and Systems FOR COMMON HEALTH ISSUES}

In this section, we first list the definitions of some healthrelated terms in Table I. Then, we review and summarize the typical objectives and applications of mHealth Sensing apps surrounding seven common health issues (i.e., depression and anxiety, sleep quality and insomnia, diabetes, heart, elder-care, diet management, tinnitus, and COVID-19) respect to the two sensing objectives (i.e., (a) Personalized Medicine and (b) Population Health).

In general, as shown in Table II, for Personalized Medicine objective, apps adopt Personal Sensing paradigm and focus on individual's health benefit via D\&Is of health status monitoring, recognition, and intervention; while for Population Health, the apps in a Crowd Sensing manner mainly aim to measure/understand population health status and discover knowledge for health benefit of populations. For example, personal mental health apps are mostly on delivering interventions by monitoring and predicting abnormal health progression for users suffering mental illnesses [68], [125]; while works on public mental health are on screening population mental status [45], [71], [126], and inferring/verifying the determinants of depression and anxiety based on massive sensing data collected from a large group of participants [72], [73]. Here we discuss the applications on the issues of depression and anxiety, sleep quality and insomnia, and Mobile Sensing in COVID-19 era as cases to study both Personal Sensing for Personalized Medicine and Crowd Sensing for Population Health perspectives.

\section{A. Depression and Anxiety}

Depression and anxiety are the main disorders in the mental health domain, which are broadly experienced by 548 million people worldwide who hardly access effective treatment [127], [128]. mHealth Sensing gives ubiquitous and flexible solutions on both sides of personal mental health management [129][132] and population mental health surveying and understanding [133], [134].

a) Personal Depression and Anxiety Monitoring and Intervention: mHealth Sensing techniques are providing broadly accessible services for individuals with mental disorders, as they can collect user's various indicators anytime and anywhere, as well as deliver timely initial diagnosis and interventions though without scarce clinical resources [17]. A typical
TABLE I: Terms and Definitions

\begin{tabular}{|c|c|}
\hline Terms & Definitions \\
\hline Mobile Health & $\begin{array}{l}\text { Namely mHealth, a term used for the practice of } \\
\text { medicine and public health supported by mobile } \\
\text { devices [55] }\end{array}$ \\
\hline Personal Sensing & $\begin{array}{l}\text { A technique collecting and analyzing data from sen- } \\
\text { sors embedded in the context of individual's life with } \\
\text { the aim of identifying his/her behaviors, thoughts, } \\
\text { feelings, and traits [56] }\end{array}$ \\
\hline Crowd Sensing & $\begin{array}{l}\text { A technique where a large group of individuals hav- } \\
\text { ing mobile devices capable of sensing and comput- } \\
\text { ing collectively share data and extract information } \\
\text { to measure, map, analyze, estimate or infer (predict) } \\
\text { any processes of common interest [57] }\end{array}$ \\
\hline Passive Sensing & $\begin{array}{l}\text { Sensing via devices that detect and respond to some } \\
\text { type of input from the physical environment [58] }\end{array}$ \\
\hline Health Status & $\begin{array}{l}\text { One's medical conditions (both physical and men- } \\
\text { tal), claims experience, receipt of health care, medi- } \\
\text { cal history, genetic information, evidence of insura- } \\
\text { bility, and disability [59] }\end{array}$ \\
\hline Health Outcomes & $\begin{array}{l}\text { Health events occurring as a result of interventions } \\
{[60]}\end{array}$ \\
\hline Health Determinants & $\begin{array}{l}\text { Conditions which contribute to a wide range of } \\
\text { health and quality of life-risks and outcomes [61] }\end{array}$ \\
\hline Mental Health & $\begin{array}{l}\text { A state of well-being in which the individual real- } \\
\text { izes his/her own abilities, can cope with the normal } \\
\text { stresses of life, can work productively and fruitfully, } \\
\text { and is able to make a contribution to his/her commu- } \\
\text { nity [62] }\end{array}$ \\
\hline Treatment Effects & $\begin{array}{l}\text { Causal effect of a given treatment or intervention on } \\
\text { an outcome variable of health interests [63] }\end{array}$ \\
\hline Health Benefit & $\begin{array}{l}\text { Positive phenomenons that a medicine treatment, } \\
\text { substance or activity is improving health [64] }\end{array}$ \\
\hline Health Intervention & $\begin{array}{l}\text { A treatment, procedure, or other action taken to } \\
\text { prevent or treat disease, or improve health in other } \\
\text { ways [65] }\end{array}$ \\
\hline Digital Biomarkers & $\begin{array}{l}\text { Objective, quantifiable physiological and behavioral } \\
\text { data that are collected and measured by means of } \\
\text { digital devices [66] }\end{array}$ \\
\hline
\end{tabular}

application of Personal Sensing for depression and anxiety is Mobilyze! [68], a mobile mental intervention application with a technical framework including context sensing and ecological momentary intervention modules. Specifically, by collecting contextual data such as locations, recent calls, ambient light, and other 38 settings and feeding them into a medical diagnosis model, it infers user's mental health status; then, the app provides interventions such as message notifications to guide users to deal with specific contexts (e.g., lack of motivation or communication). Furthermore, in personalized mental health domain, the concept of just-in-time adaptive intervention (JITAI) was proposed to guide timely and personalized interventions [135]. For example, advances in artificial intelligence are promoting smarter decision-making of when and where it is most helpful to provide supportive interventions by learning with individual's historical behaviors [136], [137].

b) Population Depression and Anxiety Survey: Mobile Sensing techniques are increasingly being adopted to population depression and anxiety surveys, as they provide a low-cost (both in labor and time), widespread, and online data collection manner versus laborious and high-cost clinical testing and ques- 
TABLE II: A summary table of the mHealth apps on seven common health issues created for the motivations in (a) Personalized Medicine and (b) Population Health respectively

\begin{tabular}{|c|c|c|c|c|}
\hline \multirow{2}{*}{ Health Issues } & \multicolumn{2}{|c|}{ Personalized Medicine } & \multicolumn{2}{|l|}{ Population Health } \\
\hline & Objectives & Applications & Objectives & Applications \\
\hline $\begin{array}{l}\text { Depression } \\
\text { and Anxiety }\end{array}$ & $\begin{array}{l}\text { Self-identifying and reducing } \\
\text { depression and anxiety }\end{array}$ & $\begin{array}{l}{[67],[68],} \\
{[69],[10],} \\
{[70],[45]}\end{array}$ & $\begin{array}{l}\text { Population mental health screening } \\
\text { and determinants inferring }\end{array}$ & $\begin{array}{l}{[71],[72],} \\
{[49],[73],} \\
{[74]}\end{array}$ \\
\hline $\begin{array}{l}\text { Sleep Quality } \\
\text { and Insomnia }\end{array}$ & $\begin{array}{l}\text { Monitoring and interventions } \\
\text { to promote sleep quality }\end{array}$ & $\begin{array}{l}{[75],[43],} \\
{[76],[9]}\end{array}$ & $\begin{array}{l}\text { Population sleep statistics for } \\
\text { understanding sleep science issues }\end{array}$ & {$[77],[78]$} \\
\hline Diabetes (type 2) & $\begin{array}{l}\text { Glucose monitoring for type } \\
2 \text { diabetes management }\end{array}$ & $\begin{array}{l}{[5],[79],} \\
{[80],[81]}\end{array}$ & $\begin{array}{l}\text { Understanding the social determinants } \\
\text { contributing to diabetes }\end{array}$ & {$[82],[83]$} \\
\hline Heart & $\begin{array}{l}\text { Heart rate monitoring and } \\
\text { heart disease prevention }\end{array}$ & $\begin{array}{l}{[84],[85],} \\
{[86],[87],} \\
{[88],[89]}\end{array}$ & $\begin{array}{l}\text { Researching the impact of determinants } \\
\text { on cardiovascular diseases }\end{array}$ & {$[90]$} \\
\hline \multirow[t]{2}{*}{ Elder-care } & $\begin{array}{l}\text { In-home care service and } \\
\text { assistance }\end{array}$ & {$[91],[92]$} & \multirow{2}{*}{$\begin{array}{l}\text { Understanding the health status and } \\
\text { lifestyle of the elderly population }\end{array}$} & \multirow[t]{2}{*}{ [93] } \\
\hline & $\begin{array}{l}\text { Outdoor monitoring and } \\
\text { notification }\end{array}$ & [94], [95] & & \\
\hline $\begin{array}{c}\text { Diet and Weight } \\
\text { Management }\end{array}$ & $\begin{array}{l}\text { Diet self-monitoring and } \\
\text { exercise management }\end{array}$ & $\begin{array}{l}{[52],[53],} \\
{[83],[96]}\end{array}$ & $\begin{array}{l}\text { Understanding population eating } \\
\text { patterns, episodes, and disorders }\end{array}$ & [97] \\
\hline Tinnitus & $\begin{array}{l}\text { Tinnitus self measurement } \\
\text { and retraining therapy }\end{array}$ & [98], [99] & $\begin{array}{l}\text { Studying symptoms, causes, and } \\
\text { treatments of tinnitus population }\end{array}$ & $\begin{array}{l}{[100],[101],} \\
{[102],[103]}\end{array}$ \\
\hline \multirow[t]{2}{*}{ COVID-19 } & Automatic self-diagnosis & $\begin{array}{l}{[104],[105],} \\
{[106],[107]}\end{array}$ & $\begin{array}{l}\text { Population screening the spread of } \\
\text { COVID-19 }\end{array}$ & $\begin{array}{l}{[108],[109],} \\
{[110],[111]}\end{array}$ \\
\hline & $\begin{array}{l}\text { Contact tracing for infectious } \\
\text { risk estimation }\end{array}$ & $\begin{array}{l}{[112],[113],} \\
{[114],[115]}\end{array}$ & $\begin{array}{l}\text { Public Health Policy Evaluation and } \\
\text { Development }\end{array}$ & $\begin{array}{l}{[116]-[118],} \\
{[119]-[121],} \\
{[122]-[124]}\end{array}$ \\
\hline
\end{tabular}

tionnaires. For example, by studying the correlation between anxiety and behavioral indicators (e.g., activity locations, text messages, and calls) in a 54-students group over two weeks, Boukhechba et al. [73] proposed flexible anxiety assessment methods for monitoring college students via mobile phone apps.

c) Population Mental Health Determinants Understanding: New inspirations and knowledge about population mental health determinants can be gained via massively collecting and comparatively analyzing data among populations [71], such as causal inference between social anxiety and group behavioral patterns [138]. For example, a Mobile Crowd Sensing platform - Sensus [2] was leveraged by Chow et al. to verify clinical models of depression and anxiety [72]. Taking the levels of depression and social anxiety as moderators, researchers tested the relations between state effect and time spent at home of 72 recruited students, and finally, they gain an understanding that actual home-stay is related to affect to symptoms of depression and anxiety (i.e., people with higher depression and anxiety within a day would be likely to stay at home longer in the following day).

\section{B. Sleep Quality and Insomnia}

mHealth sensing applications are widely applied to monitor sleep status, measure sleep quality, and study scientific questions in the field of sleep science. The basis for this is that the digital biomarkers (e.g., heart rate and sound of snoring) related to sleep can be easily collected by mobile sensors [139].

a) Personalized Sleep Monitoring and Insomnia Assistance: mHealth Sensing apps for personalized sleep monitoring are giving precise and trustworthy sleep quality monitoring and sleep-aid services to users [9], [75], [139]. Several sleep monitoring systems are deployed on wearable devices requiring users to wear a product embedded with specific sensors during sleeping, which is either limited in clinical environment [140] or uncomfortable to the users [141]. A new trend in mHealth Sensing for sleep monitoring is using off-the-shelf mobile phones built-in sensors such as microphones and accelerometers to detect the sleep duration and infer sleep quality. For example, Hao et al. [43] proposed to leverage microphone to detect the events closely related to sleep quality such as ambient noise, body movement, and sound of snoring [75] to enable personalized and in-place sleep quality monitoring. Furthermore, $\mathrm{Gu}$ et al. [76] mined the sleep stage (e.g., week sleep, deep sleep, and rapid eye movement) by detecting the sleep stage transition integrating mobile-sensed sleep environment and personal factors by a statistical model, which provides finegrained descriptions of sleep status.

b) Population Sleep Science Research: Crowd Sensing apps are widely used to create population sleep status datasets for the researches in sleep science, such as understanding the issues on psychological research and sleep science, as sleep may contribute to or be affected by one's lifestyle and psychological conditions. In practice, to understand the behavioral pattern between phone usage and sleep quality, recently, Sharmila et al. [77] collected a large-scale phone usage dataset and sleep questionnaires from 743 participants of different ages and socioeconomic backgrounds in a Crowd Sensing manner and figure out the effect of mobile phone usage patterns on sleep using statistical methods. Additionally, Abdullah et al. [78] proposed to study the effects of sleep quality on people's daily rhythm and well-being including levels of alertness, productivity, physical activity, and even sensitivity to pain.

\section{Mobile Sensing in COVID-19 Era}

The mobile devices that people carry around are like "witnesses" to the spread of the epidemic, as the spread of the COVID-19 virus is accompanied by human mobility and contact, where Mobile Sensing have shown its great power in 
COVID-19 era [142]; the typical contributions include personal diagnosis [104], infection traceability [112], transmission interpretation [110] and policy decision-making [143], etc.

a) Personal Automatic Self-Diagnosis via Sounds: Mobile microphones collect audio signals including sighs, breathing, heart, digestion, vibration sounds on body, which can serve as the indicators to diagnose lung diseases [144], giving great possibilities of automatic detection and diagnosis of COVID19 infection [105]. For example, Brown et al. [106] explored the promise in detecting diagnostic signals of COVID-19 from voice and coughs, which also can distinguish a user who is tested positive for COVID-19 with a cough from a healthy user with a cough. In addition to voice analysis, Han et al. [107] further explored fusion strategies to combine voice and reported symptoms which yield better detection performance.

b) Personal Contact Tracing: The COVID-19 virus spreads from an infected person's mouth or nose in small liquid particles when they cough, sneeze, speak, sing or breathe [145], causing finding the contacts of positive patients is an essential task for epidemic control. Many contact tracing mobile apps are developed and deployed for privacy-preserving and comprehensive COVID-19 tracing for individual users to check their contact history with mobile phone data [112], [113], [115]. For example, Carli et al. [114] developed WeTrace, a mobile COVID-19 tracing app which detects and records one's contact with others leveraging the interaction via Bluetooth Low Energy (BTE) communication channel; and a trusted data transmission framework is proposed to balance the health and the privacy perspectives.

c) Epidemic Spreading Analysis via Human Mobility: The significant correlation between human mobility and COVID-19 infections provides guidance on investigating and understanding the spreading of COVID-19 via multi-scale human mobility data [108]-[110]. From the perspective of the human mobility research, large-scale and long-term GPS data can be used to detect high-risk regions [146], and population traveling data (e.g., Baidu Qianxi [147]) can be leveraged to analyze the spreading path between cities and countries [148]. For example, by incorporating human mobility data into epidemic modeling, Hao et al. [111] studied how the multi-scale urban human mobility impacts the spreading process at varying levels, which provides insights on making smarter policies to respond the next outbreak.

d) Public Policy Evaluation and Making: Strict infection control policies proposed by governments have been taken to limit and mitigate the fast-spreading of COVID-19, such as lock-down, travel restrictions, quarantine, social distance ban. mHealth Sensing data among populations is contributing in evaluating and making these policies [143], [149]. For example, intuitively, several of the sensing indicators among populations, such as the average time of users stay-at-home and the number of mobile devices in a public place, can be used to measure stay-at-home and social distance policy efficiency [116], [117]. Furthermore, statistical and machine learning methods can be used to estimate, simulate, and predict the effects of the policies on controlling virus spreading driven by the population data gathered in mobile devices [119], [120], [122].

\section{Discussion}

Note that, in this work, we review and summarize the works on mHealth Sensing Apps and Systems that deployed over massive smartphones and commodity interactive devices, such as tablets, smartwatches, and other wearable consumer electronics in non-invasive sensing manners. Many other works intending to monitor physiological status of patients for medical purposes or professional devices/systems for critical cares/assisted living, such as medical sensors [150]-[154], Internet of Medical Things (Medical IoTs) and Medical Cyber-Physical Systems (Medical CPSs) [155]-[177], and medical robots [178]-[183], are not included here.

\section{TAXONOMY SyStem I: ClasSifiCATION OF MHEALTH SENSING By SENSING ObJeCTIVES}

In this phrase, we introduce the proposed mHealth Sensing taxonomy system I from sensing objectives perspective. We specify and classify major sensing objectives of mHealth Sensing apps as (a) Personalized Medicine and (b) Population Health apps. Then we further discuss the detail sensing $o b$ jectives in each step of the life cycle framework of mHealth Sensing, as shown in Figure 2.

\section{A. Objectives in Sensing Task Creation \& Participation}

The main objectives in Sensing Task Creation \& Participation step are creating tasks for specific health issues via deployed apps, then prompt the participation of the users or recruited participants to finish the sensing tasks. Since the health benefit for the participants in the two types of apps are varying (i.e., participants in Personalized Medicine apps obtain direct personal health benefit, while participants in Population Health practices hardly obtain health benefit equaling to their efforts), where the detail objectives in this step can be distinguished as service provision for personalized medicine seekers and recruitment for population health participants.

- Service Provision for Personalized Medicine Seekers Sensing apps for Personalized Medicine provide timely and accurate individual health status monitoring and personalized interventions or treatments, which can be concluded as healthcare services provision [135], [184]. In most of the Personalized Medicine cases, participants actively engage in the sensing task for personalized medicine with an expectation to seek and extend personal health benefit [185]. To this end, the detail objective of personalized medicine apps in this step is to provide exact healthcare services (e.g., exercise reminders and userfriendly interface) suiting the users' health interests and improve service quality (e.g., optimizing reminder times with algorithms) to guarantee and enhance users' active engagement [186].

- Recruitment Provision for Population Health Participants - Population Health apps are for studying population health issues from massive collected data from a large group of participants, causing a problem for participants is that - as essentially a data collection activity rather than a health service, Crowd Sensing practices are difficult to provide participants direct and sufficient health 


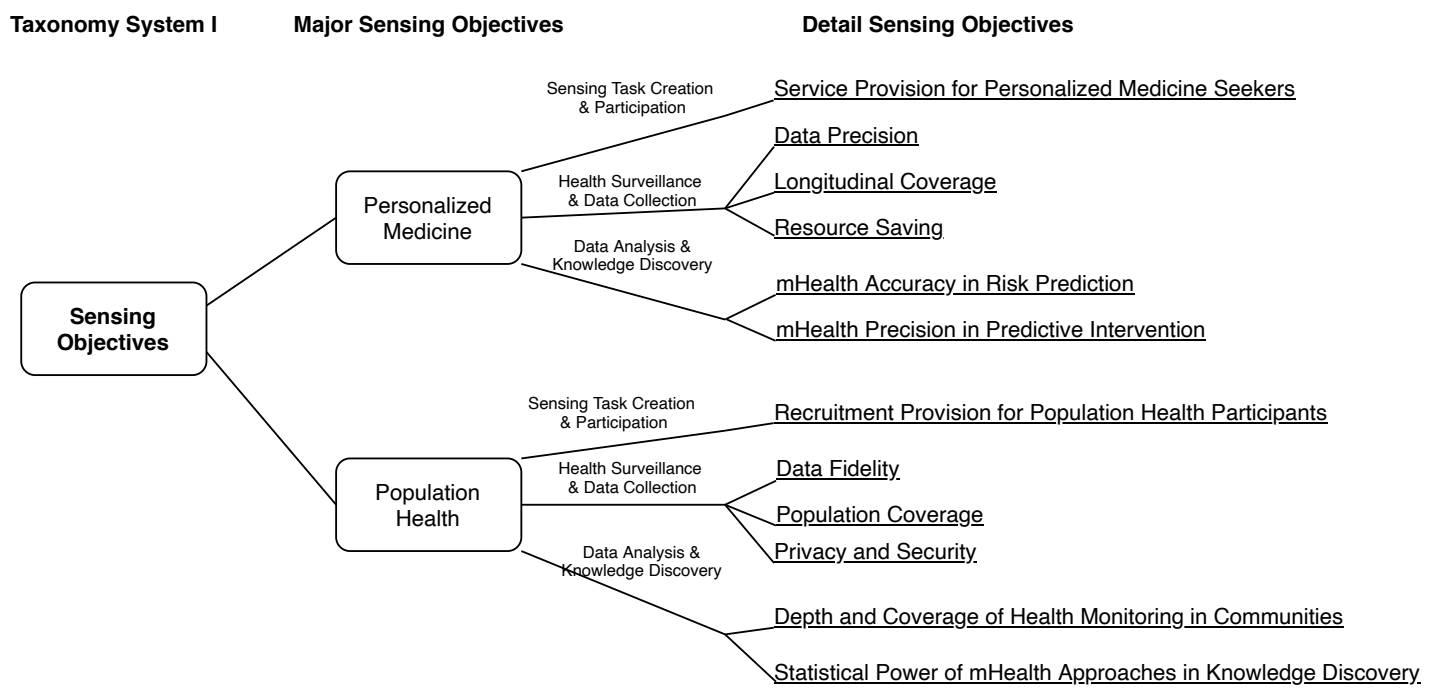

Fig. 2: Taxonomy System I - Sensing Objectives

benefit compensating for their costs and concerns (e.g., time consumption, privacy exposure [187], and battery drainage [188]). For example, in an unofficial COVID19 infectious population screening [189] or a rare clinical disease causes understanding program [190], the results are valuable for the organizers but limited for participants. The above reasons lead to a unique detail objective of Population Health apps in this step - providing recruitment to gather participants and motivating their performance with incentives [48], [191].

\section{B. Objectives in Health Surveillance \& Data Collection}

With exact sensing tasks and a pool of users/participants, the bottleneck of the next step (i.e., Health Surveillance \& Data Collection) is - how to effectively collect and gather trustworthy sensing data, with taking users' costs and concerns into account. As shown in Figure 3, we summarize that mHealth Sensing apps' trustworthiness lies on data quality and data quantity; further, the data quality can be further divided into data precision and data fidelity, and the data quantity can be divided into longitudinal coverage and population coverage. In addition, some objectives for costs and concerns aspect including resources saving and security and privacy are also crucial detail objectives. For the Personalized Medicine and Population Health apps, in practice, the detail objectives vary [192], where we discuss in the following.

a) Personal Sensing for Personalized Medicine: In apps for Personalized Medicine, to provide timely and adaptive healthcare services based on precise and sufficient data, the detail objectives of Personal Sensing in this step are data precision and longitudinal coverage for apps' trustworthiness, and resources saving in data collection process.

- Data Precision - The data precision is the most straightforward pursuit of Personal Sensing tasks, which determines the service quality of Personalized Medicine. Here we give the mobile medical devices in the intensive care unit (ICU), which is the last barrier to save the lives of dying patients in the hospital, as examples [193],
[194]. The personal wearable devices with incentive body sensors, light and sound sensors, and others precisely collecting the physical and environmental context data from ICU are typical Personal Sensing schemes with high sampling precision, finely sensing physical and environmental biomarkers such as facial expressions, functional status entailing extremity movements and postures, and environmental indicators for ICU's context [195].

- Longitudinal Coverage - It is crucial for Personalized Medicine apps to collect one's longitude health-related data with high granularity targeting at individuals. The reason behind it is two-fold: not only longitude and sufficient moment-to-moment data sampling is helpful for training a personalized model capturing the variable dynamic of health status to achieve meaningful status recognition and prediction [11], [196]-[198], but also the analyzing of the onset of some diseases is not trivial, as the determinants of diseases contribute to the onset in a long time period (e.g., monthly and annually), and there may be a time delay from contacting with triggers to the onset (e.g., an infectious disease having incubation period), which cannot be captured in a short-term observation manner [199][201].

- Resources Saving - Keeping mobile data sampling causes severe battery, hardware, and computing resources consumption of the mHealth Sensing devices, especially for most Personalized Medicine apps whose data collection actions are generally continuous and intensive [202], [203], which is a big concern of users. Thus, resources saving measures are in crucial need to lower the cost of mHealth apps. Specifically, the type of sensors and the rate of sampling correlated to data accuracy and sampling abundance should be considered to save costs. For example, Ben et al. [204] proposed SenseLess, an energyefficient optimization strategy to maximize the battery life by using resource-constraint sensors whenever possible, thereby to use resource-consuming sensors at a low frequency. In addition to the above, some insights from data 


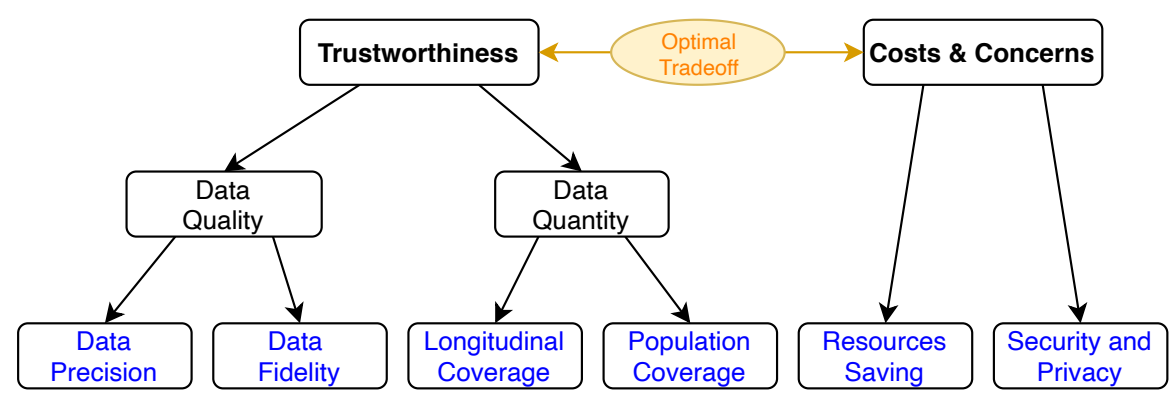

Fig. 3: Detail sensing objectives in Health Surveillance \& Data Collection

science such as data inference [205], and data transferring [206] also enable mHealth Sensing systems to collect partial data but work efficiently.

b) Crowd Sensing for Population Health: In Population Health practices, the task of Health Surveillance \& Data Collection is to build a large-scale and error-free data pool surrounding the health issues to be analyzed and researched, with detail objectives of ensuring data fidelity and enlarging population coverage in the sensing process, as well as releasing users' concerns such as privacy and security.

- Data Fidelity - Versus data precision, data fidelity in the mHealth Sensing context refers to that there is no human error (e.g., intentional cheating or equipment failure) in the gathered data [207]-[209]. For example, some participants may deliberately misreport data in order to obtain illegal rewards, which is disastrous for the Population Health mission. Especially, different from the collections of some general datasets (e.g., traffic speed data or urban temperature data) which can be gathered in a short time, collecting health-related data requires enormous manpower, incentive cost, and devices resources in a long time; once there are human errors in the data pool, it would lead to biased health modeling, inaccurate treatment effects, and wrong medical conclusions, which are harmful to the health and well-being purposes.

- Population Coverage - Enlarging the population coverage of Health Surveillance \& Data Collection is beneficial to obtain statistical-significant and generalized Data Analysis \& Knowledge Discovery. Specifically, in Crowd Sensing paradigm, some general guarantees for population coverage are age, gender, region, patient groups coverage; for varied research purposes, the coverage requirements for population attributes vary [210], [211]. For example, data for population mental health researches should cover balanced genders and diversified ages for comparative analysis and knowledge discovery with no/limited prior knowledge leveraging machine learning [212] or statistical inference [213] approaches; data for sleep science researches should cover kinds of patient groups such as sleep apnea, insomnia, Parkinson disease, and PLMD, as well as healthy patients as control group, which is conducive to train universal sleep models performing well in all populations with statistical power ensured.

- Privacy and Security - The risk of privacy leakage in Population Health apps is greater [214], [215], as it re- quires regular sensitive health-related data uploading and offloading between mobile devices and cloud servers via networks in practice, thus the privacy and security issues are big user concerns [216]. To be specific, as far as what can be concerned in Crowd Sensing data collecting and uploading process, privacy problems can be generalized and classified as identity privacy [217] (participants do not want to expose personal information), data privacy [218] (health-related data is top sensitive), attribute privacy [219] (for attributes such as locations and trajectories), task privacy [220] (the sensing tasks may correlate to participants' illnesses), and decentralized privacy [221] (frequent communication with a central server could be hacked). Note that privacy and security have been widely studied in Medical IoTs or Medical CPSs [163], [164], [168]-[170], [222], [223]. Compared to medical IoT or medical CPS deployed at homes or professional clinics, the mHealth sensing systems leveraging the sensors deployed at ubiquitous mobile devices make the privacy and security issues even more complicated.

The truthworthiness mostly gained by intensive, longitude, and broad-coverage data sampling of users/participants, while the costs \& concerns of users generally caused by the above requirements, which leaves app developers to make an optimal tradeoff between the two objectives in practice, as shown in Figure 3. On the one hand, the developers should design and develop the apps with verification that minimizes data access privileges subject to the actual needs, to release the costs \& concerns. On the other hand, advances in resources saving and privacy protection approaches may make it possible for developers to obtain additional permissions from users, which further improves the apps' truthworthiness.

In addition, though the detail sensing objectives in Health Surveillance \& Data Collection step are specified as the above perspectives, these objectives are usually overlapped. For example, data precision and longitudinal coverage are also meaningful in Crowd Sensing for Population Health practices, but compared to these two objectives, data fidelity and population coverage are in need of relatively dedicated D\&Is for specific existing problems.

\section{Objectives in Data Analysis \& Knowledge Discovery}

After gathering expected high-quality personal or population data pools, the main objective in Data Analysis \& Knowledge 


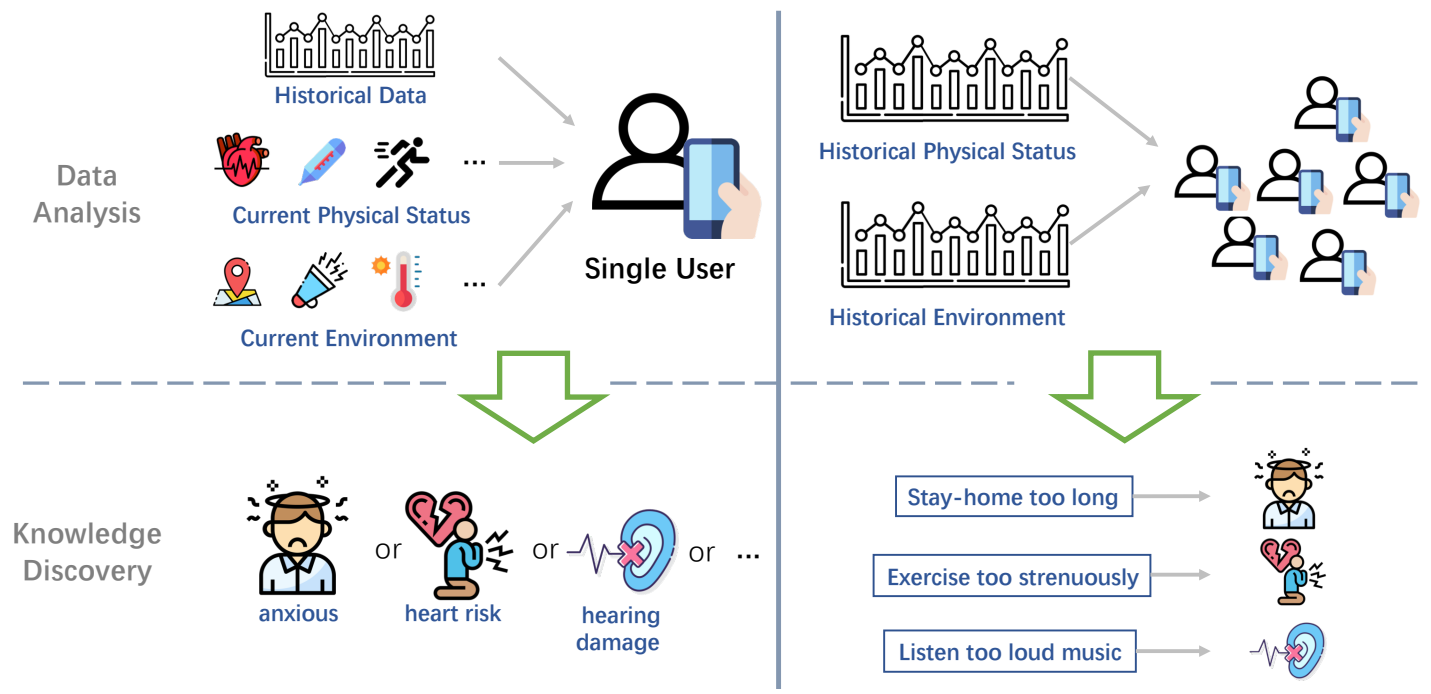

Fig. 4: The comparison of main objectives of Data Analysis \& Knowledge Discovery in the apps for Personalized Medicine and Population Health

Discovery is to make sense of gathered data to discover healthrelated knowledge about individuals and populations via data analysis approaches, and provide effective and timely healthcare services or feedback [224], [225].

a) Personal Sensing for Personalized Medicine: Apps for Personalized Medicine usually recognize [226] or predict [227], [228] individual user's health status by integrating his/her historical, as well as current physical and environmental data surrounding a specific health issue to accurately recognize/predict health risks and provide precise healthcare interventions at the right time, as shown in Figure 4.

- mHealth Accuracy in Risk Prediction - Effective personalized health services rely on the accuracy in the status modeling and risk prediction based on collected data. Sufficient multimodal data throughout user's life such as self-reported medical history, physical biomarkers (e.g., heart rate, body temperature, and moving speed), and environmental biomarkers (e.g., points of interest, locations, temperature, surrounding noises) provides great potential in accurately modeling and predicting one's health outcomes and progressions of both physical and mental via machine learning approaches [229]-[231]. For example, by passively monitoring schizophrenia patients' psychiatric symptoms represented by 7-item scale scores and behaviors and contextual characteristics (e.g., physical activity, conversation, mobility) over a period ranging from 2-12 months, Wang et al. [232] proposed a prediction system which predicts psychiatric symptoms' dynamics and progression solely based on mHealth Sensing data without traditional self-reported ecological momentary assessment (EMA) methods.

- mHealth Precision in Predictive Intervention - A typical detail objective in this step for Personalized Medicine apps is to provide predictive interventions with high mHealth precision responding to recognized/predicted health outcomes and progressions (e.g., increasing depression and anxiety, exposing to high heart risk, and being damaged hearing). Specifically, the precision above lies on precise intervention timing, measures, and intensity, which leads to just-in-time, adaptive, and effective mHealth supporting services [135], [233]. For example, Costa et al. [130] proposed to improve one's cognitive performance by unobtrusively regulating emotions with smartwatch notifications in varying detected heart rates. Lei et al. [234], by formulating the intervention tasks in real-time as a contextual bandit problem, provided an online actorcritic algorithm as an intervention strategy to guide JITAI practices.

b) Crowd Sensing for Population Health: Crowd Sensing practices investigate population health issues by comprehensively mining massive health-related data among researched groups such as monitoring and screening the population health status in a region in both depth and coverage [231], and verifying [77] and inferring [74] the determinants of specific diseases via powerful statistics-based approaches.

- Depth and Coverage of Population Health Monitoring in Communities - In Population Health apps (especially for the apps on population health monitoring, screening, and surveying), in terms of data analysis, it is meaningful to deeply mine and widely enlarge the information of targeting communities leveraging collected Crowd Sensing data. For example, in many mHealth Crowd Sensing practices, some specific characteristics of health problems (e.g., the contact infection of infectious diseases [235], familial heredity phenomenon of genetic diseases [236], and regional relevance of conventional health habits [237]) give great possibility to finish a mobile population health screening of the whole community by only investigating a subset of this group, which is a manner with accuracy guarantee and lower cost. 


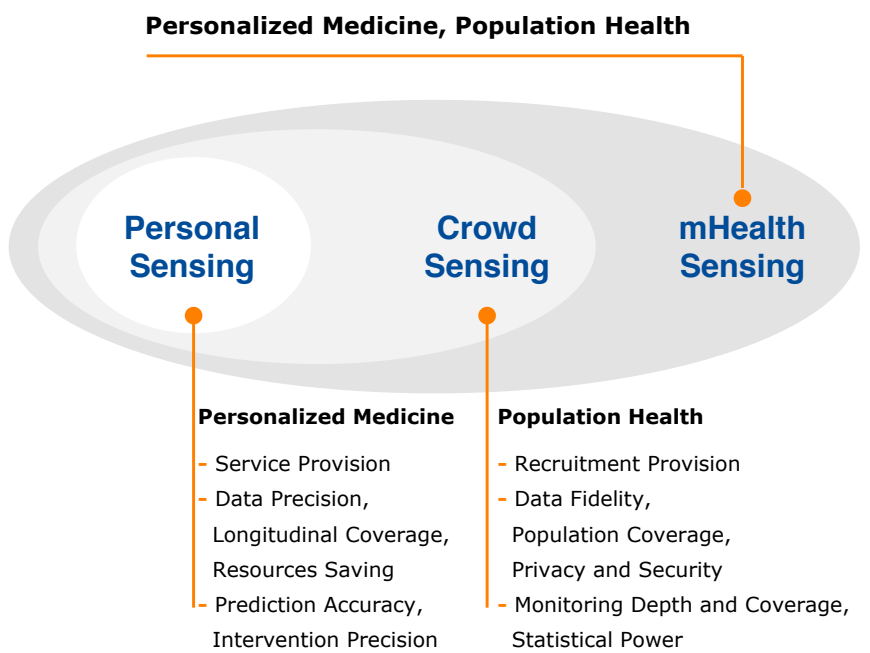

Fig. 5: The relationship between the objectives of Personal Sensing and Crowd Sensing paradigms

- Statistical Power of mHealth Approaches in Knowledge Discovery - The statistical power of the mHealth approaches is a key pursuit for medical-related knowledge discovery in large-scale population data. Specifically, "From data to knowledge" is a prevailing topic in the data science field; in mHealth field, Crowd Sensing is being used as a useful tool to collect and analyze massive population health-related data to obtain medical knowledge, where new knowledge can be summarized or inferred by statistical methods for a better understanding of health determinants [61], such as staying home too long causes mental health problems [73], lacking exercise would increase the risk of heart attack [86], and listening too loud music leads to tinnitus [99]. For example, Zhang et al. [211] revealed how human mobility features extracted from large-scale human mobility data affect one's health conditions and which group of features contribute significantly leveraging statistical approach - shapely additive explanation value analysis, which shed light on how to understand human mobility data in health monitoring.

It is worth mentioning that, deploying Crowd Sensing devices can be regarded as a accumulation of the number of Personal Sensing devices deployed in a community. Thus, most of the objectives in Personal Sensing are also what the Crowd Sensing paradigm pursues in practice. To this end, in this paper we conclude the objectives of Personal Sensing, Crowd Sensing, and mHealth Sensing as shown in Figure 5, where their objectives are progressive. For example, intuitively, in Crowd Sensing practices, improvements in cost saving and data accuracy will surely also improve the performance of the apps.

\section{TAXONOMY SYSTEM II: Classification OF mHealth Sensing by Sensing Paradigms and D\&Is}

With respect to distinguished sensing objectives (i.e., Personalized Medicine and Population Health) and their details discussed in Section III, two sensing paradigms (i.e., Personal Sensing and Crowd Sensing) are correspondingly proposed to deal with related technical issues through detailed D\&Is. In this phrase, as shown in Figure 6, for each step of mHealth
Sensing life cycle, varying detail D\&I issues of the two sensing paradigms are discussed.

\section{A. Design \& Implementation Issues in Sensing Task Creation \& Participation}

To prompt the users' participation and task execution leveraging services and recruitment respectively for Personal Sensing for Personalized Medicine and Crowd Sensing for Population Health apps discussed in III-A, in this section, we intend to specify the detail D\&I issues of the two paradigms as followings.

a) Personal Sensing for Personalized Medicine: The promotion of user engagement in Personalized Medicine apps is by providing services. Here we discuss two typical forms of user engagement services - clinical health service and humancomputer interaction $(\mathrm{HCI})$ and gamification and attraction in detail.

- Clinical Health Service and HCI - Providing straightforward and effective clinical health service with good HCI design for user experience is the most intuitive way to increase users' active engagement, since the essential motivation of the users downloading the app is to obtain personal health benefit [238], [239]. In practice, user engagement strategies can be organized as setting sensing health-related targets around users' personalized objectives, delivering adaptive therapeutic feedback including positive reinforcement, reflection reminders, and challenging negative thoughts [240], [241], and designing easyto-use platforms [188]. For instance, Rabbi et al. [242] designed an app named SARA, which integrates engagement strategies including contingent rewards, badges for completing active health tasks, funny memes/gifs \& lifeinsights, and health-related reminders or notifications.

- Gamification and Attraction - Gamifying the mHealth Sensing apps for providing entertainment would promote user engagement, as not only the mobile sensing data can be used as input for gamification [243], but also mobile apps are excellent and prevailing mobile carriers for pervasive entertainment [244]. In practice, gamification strategies are widely applied throughout the life cycle of Personalized Medicine apps to promote participation, such as self-report data collection [242], [245] (e.g., setting the goals of the game as the indices to be sensed), data preanalysis on client [246] (e.g., pop-up windows asking the user about the activity and status when the app detects a sequence of abnormal indices), and health intervention wrapping [247] (e.g., relaxing users under depression via games). Typically, Lee et al. [248] revealed the effects of gamification in increasing user engagement for mHealth apps and how it works differently according to health status, age, and gender, which gives insights on sustained health maintenance when designing mHealth Sensing services.

b) Crowd Sensing for Population Health: Though participants in Population Health tasks may also actively/voluntarily engage attracted by D\&Is for services (i.e., services and HCI designs) above [249], a crucial problem in the tasks does exist - 
Taxonomy System II Sensing Paradigms Detail Design and Implementation Issues

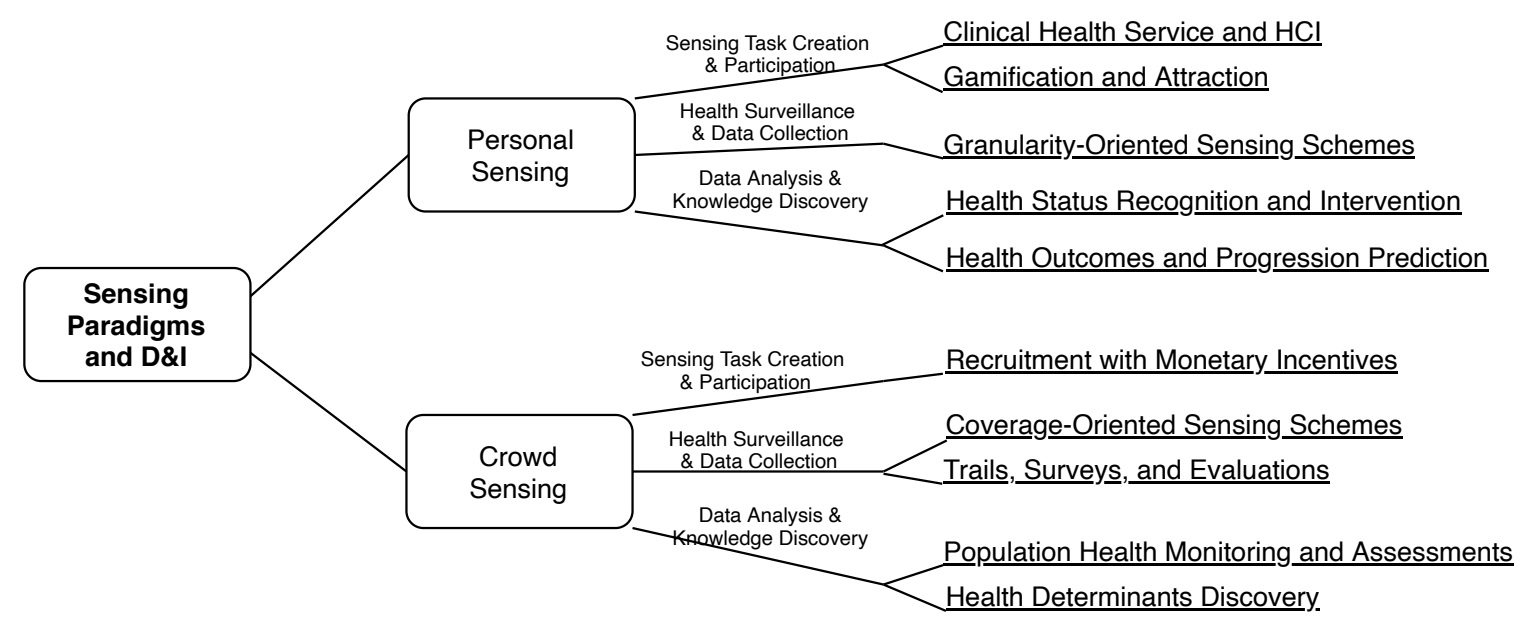

Fig. 6: Taxonomy System II - Sensing Paradigms and D\&Is

participants may not obtain direct/enough health benefit compensating their efforts, leading to its unique incentive mechanisms - recruitment with monetary incentives [38]. Worth mentioning, in most Crowd Sensing for Population Health practices, the incentive mechanisms (i.e., services and recruitment) are not used strictly separated; they can be wrapped together to optimize the incentive effects [39], [250], [251].

- Recruitment with Monetary Incentives - Monetary incentivization is an intuitive strategy to quantify and equalize participants' efforts and benefits, which suits to prompt participation in Crowd Sensing tasks (though some voluntary Crowd Sensing activities also do exist). The monetary incentives strategies can be further divided into categories as platform-centric and user-centric methods [48]. Specifically, platform-centric methods refer to that the number, allocation, and adjustment of monetary rewards are charged by the organizer. For example, through some optimized incentive strategies based on game theory and statistics [252], the organizer leads the task and adjusts the strategy by measuring the individual/overall performance of the participants [253]. The user-centric methods are conducted in an auction manner, in which, generally, users bid for the sensing task published by the organizers, and the subset of participants with the lowest bid is dynamically allocated to complete the sensing tasks within minimal cost [254].

In addition to the above incentive models, there are some works focusing on the incentive allocation problems [47], [51], [255]-[259] under certain budgets and data collection objectives/constraints, especially for environment monitoring applications. Specifically, Xiong et al. proposed several participant recruitment strategies [51], [255]-[257] for mobile crowd sensing in either online or offline manners. Wang et al. [258], [259] studied the problem of participant recruitment and task/incentive allocation in the context of multi-tasking, where incentives are allocated to the same pool of potential participants for multiple tasks with shared budgets, via hierarchical data collection objectives. The same group of researchers

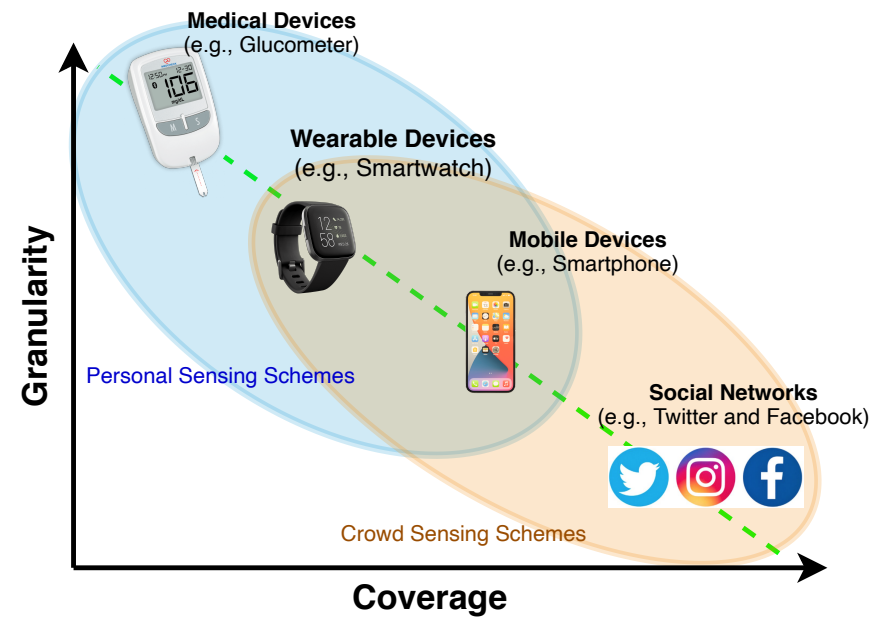

Fig. 7: The comparison of sensing schemes from granularity and coverage perspectives

also studied to collect population health-related data from large crowds with non-monetary incentives in practice [54], [260].

\section{B. Design \& Implementation Issues in Health Surveillance \& Data Collection}

In Health Surveillance \& Data Collection step, for the objectives of data quality, sensing schemes and data gathering approaches are the main D\&I issues. For example, some objectives on data quality (i.e., data accuracy and population coverage) depend on sensing schemes (e.g., sensors and devices), which have varying emphases (i.e., granularity and (population) coverage) in D\&Is. As shown in Figure 7, in mHealth Sensing field, either it needs widespread devices (e.g., mobile devices and social network) with pervasive coverage among populations, or it needs dedicated devices (e.g., portable medical devices) for accuracy and professionalism. However, limited by the costs and the accessibility of unique devices to data in different contexts, it is hard to trade off granularity and coverage in practice; for example, it is difficult for a device being used to sense contexts with high granularity requirements 
to have good coverage in populations, making it not trivial to be used as Crowd Sensing schemes.

a) Personal Sensing for Personalized Medicine: Though the two paradigms sometimes use common sensing schemes (e.g., wearable devices and mobile devices shown in Figure 7) under some circumstances, while, for the objectives on numerical accuracy and longitudinal coverage, the sensing schemes in Personal Sensing practices are granularity-oriented.

- Granularity-Oriented Sensing Schemes - To accurately monitor user's physical/environmental dynamics in a realtime manner, some dedicated and intensive sensors deployed in medical devices are commonly used in Personal Sensing practices, such as mobile fall detection devices on elderly care in daily scenarios [261], [262] and intensive location/maneuvers monitoring devices in hospital scenarios [263], [264] which are equipped with radar. For example, Fang et al. [265], [266] purposely embedded radio sensor into wearable devices as a new powerful sensing modality to provide whole-body activity and vital sign monitoring in clinical, which serves as an example that specialized sensing schemes provide richer function in Personal Sensing scenarios.

b) Crowd Sensing for Population Health: To broadly collect health-related data with guarantees of population coverage and data fidelity, in Crowd Sensing practice, the detail D\&Is lie on coverage-oriented sensing schemes (for population coverage), trials, surveys, and evaluations (for data collection efficiency and fidelity).

- Coverage-Oriented Sensing Schemes - In Crowd Sensing practices, though many sensing schemes are the same as those used in the Personal Sensing apps as shown in Figure 7, while, in order to enable the system to be used in a larger population coverage, ubiquitous sensing schemes are prevailing in Crowd Sensing practices, such as social medias (e.g., Facebook and Twitter) [267], [268] and large-scale human mobility data which is not gathered dedicatedly for health-related purposes [73], [269], [270]. For instance, Choudhury et al. used passive sensed data from social medias to measure and predict the depression in population [267], [271], even further to discover shifts to suicidal tendency from content in Reddit [272].

- Trails, Surveys, and Evaluations - In Crowd Sensing data collection process, it is essential to motivate participants to upload sensing data with efficiency and fidelity. Typically, trail and survey schemes are for the efficiency, and data evaluation schemes are for the fidelity. As for trails and surveys, micro-randomized trials (MRTs) are tools for maintaining and improving participants' efficiency by optimizing the combinations of incentives (e.g., varying levels of monetary incentives, and virtual rewards) [251], [273], [274]. With MRTs, participants first randomly grouping to collect data under varying incentives, then in the following sensing loops, the collected data in the previous round is used to measure which combinations of incentives are optimal. As for evaluation schemes, they are for enforcing data fidelity [275]. In specific, once a new round of data collected, but before accepting the data as convinced, the data fidelity is estimated and only convinced data is gathered; according to the estimation, positive or negative feedback is given to participants to reward/punish them in the following rounds. An intuitive scheme, named truth discovery [276], is to let multiple participants finish a same task to find the wrong-data providers [277]. However, this repeated validation manner cannot be adopted to health-related data collection since sensitive personal data can only be sensed by the individual himself/herself. While the trust framework [278] is an alternative means to solve this. Some measurement methods can be used to establish a credit rating measurement system for participants, and implement different acceptance of data contributed by users with different credits, and varied tasks and incentives are dynamically allocated to enforce participants' performance in the following sensing rounds [279]-[281].

\section{Design \& Implementation Issues in Data Analysis \& Knowl- edge Discovery}

With respect to the detail sensing objectives listed in Section III-C, we one-by-one discuss the detail D\&Is issues in this section. Furthermore, in order to fully study detailed technical perspectives of the two sensing paradigms, inspired by the mHealth Personal Sensing framework proposed by Mohr et al. [56], we formulate the D\&Is of Data Analysis \& Knowledge Discovery workflow as shown in Figure 8.

a) Personal Sensing for Personalized Medicine: Generally, in Personal Sensing, Data Analysis \& Knowledge Discovery serves to mine collected raw data to realize health status recognition and interventions or health outcomes and progression predictions.

- Health Outcomes and Progression Predictions - Due to the fact that the determinants of health problems are affected by multiple pathogenic factors and progress slowly, it is not trivial for conventional clinical methods to effectively predict health outcomes and progressions via clinical medical records [282]. Personal Sensing data provides rich personalized information to model the health status of user and predict his/her future health outcomes and progression. As shown in Figure 8 (a), after collecting raw data (e.g., GPS location, microphone signal, and screen status) from user, digital physical and environmental biomarkers (e.g., places, ambient noises, and app usages) can be extracted to represent user's statements [283], [284]. Then, personal health status modeling and prediction models analyze individuals' clinical status and predict health outcomes and progressions with advanced algorithms considering longitudinal user's data both in current and historical. In the machine learning era, feature embedding and deep learning techniques are good tools to solve the challenges in multidimensional pathogenic factors and long-term disease progression, respectively. Specifically, after extract behavioral/environmental features from multi-modal collected data, feature embedding (e.g., graph embedding) would automatically learn and extract influential features, and deep learning models (e.g., 


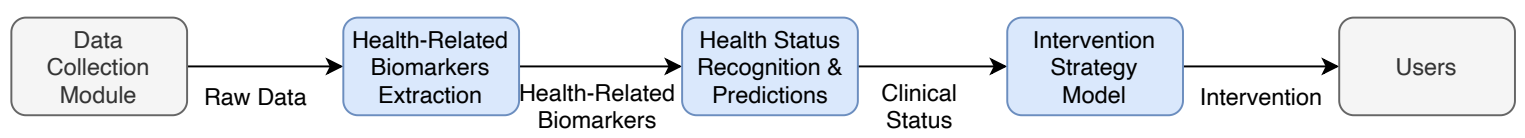

(a) Personal Sensing for Personalized Medicine - Both for Health Status Recognition \& Prediction

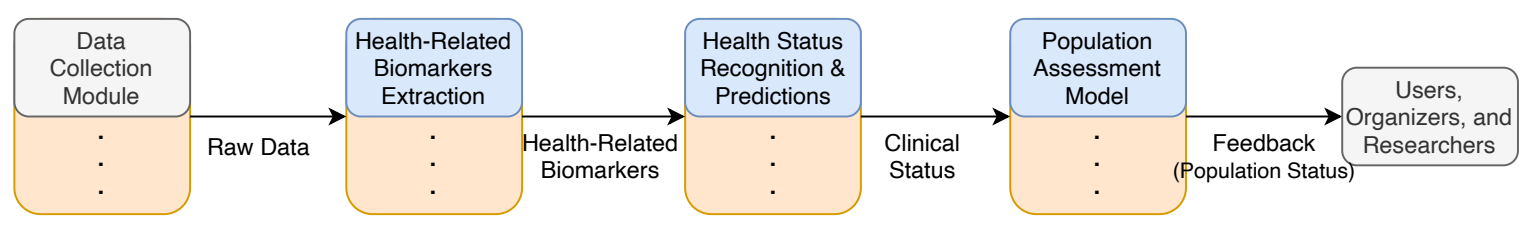

(b) Crowd Sensing for Population Health Monitoring and Assessments

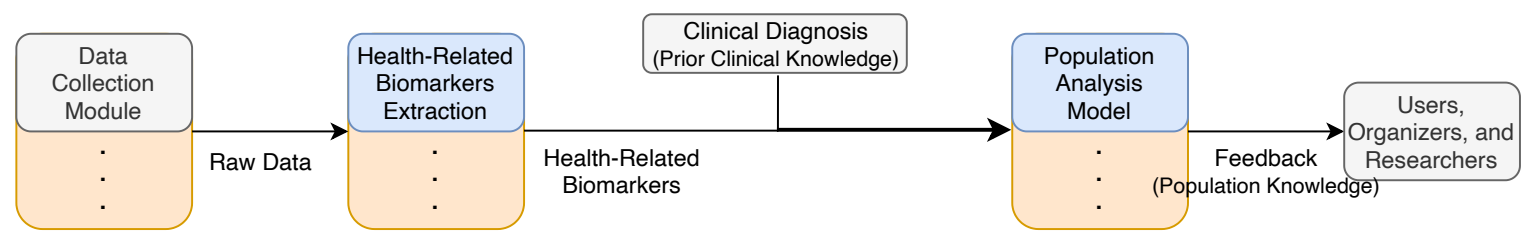

(c) Crowd Sensing for Population Health Determinants Discovery

Fig. 8: The D\&Is of Data Analysis \& Knowledge Discovery workflow in three typical Personal Sensing and Crowd Sensing apps.

RNNs, GNNs) would serve as predictors with great performance in dynamically capturing patterns in time or other dimensions [227], [228], [285], [286].

- Health Status Recognition and Interventions - As shown in Figure 8 (a), according to different health status, the Personal Sensing apps could deliver varying interventions as healthcare services for users. What's more, the apps can further recognize users' following status for measurement of the interventions' effectiveness to refine the strategies and suit the users. As for implementations, MRTs [287] are ideal tools to deliver JITAI for patients. As stated in Section IV-B, analogous to the designs of MRTs in improving data collection efficiency, it can also be formulated to optimize the effectiveness of various interventions.

b) Crowd Sensing for Population Health: We discuss two typical applications (i.e., population health status measurement and health determinants discovery) to conclude D\&I in Crowd Sensing applications.

- Population Health Monitoring and Assessments - Intuitively, as shown in Figure 8 (b), once sensing tasks among a group of users are adopted, organizers can scan the clinical status among populations and achieve and assessment of population status. Furthermore, in the population assessment models, some algorithms inspired by some characteristics of population health problems, such as spatial correlation, help achieve low-error surveys of entire target group by only monitoring a subset of users. For example, to investigate a large group of people such as citizens of a country, Chen et al. [54] studied and indicated spatio-temporal correlation of neighboring regions and proposed to do data inference for the whole map with limited region samples, which gives insights in operating population health monitoring in a Crowd Sensing manner.

- Health Determinants Discovery - What's more, as shown in Figure 8 (c), the D\&I of applications on popula- tion health determinants discovery differs. Specifically, in some clinical practices, especially for mental health and chronic illness, with prior knowledge such as clinical diagnosis and EMA test, organizers massively collecting multimodal data from participants (participants may be divided into experimental group and control group in this process) and analyze population pattern among participants' biomarkers and clinical diagnosis to understand and discover health determinants; finally, population knowledge serves as feedback, which benefits to both participants themselves (for obtaining health knowledge on health issues interested) and organizers and researchers (for new insights on the researched disease). In the implementation aspect, large-scale data analysis methods give great insights on population health knowledge discovery (e.g., inference and understanding) from Crowd Sensing data. For instance, machine learning methods such as clustering algorithms are widely used to classify individuals into groups according to common health-related patterns [288]. In addition, statistical methods such as statistical inference are also promising confirmatory tools for understanding and inference on clinical conclusion than trainingbased models with confidence intervals on assessment, which, compared with machine-learning-based methods, is commonly leveraged by clinical scientists since it is a hypothesis-driven and interpretable manner [289]. For example, Boukhechba et al. used Social Interaction Anxiety Scale (SIAS) correlation analysis to understand how social anxiety symptoms manifest in the daily lives of college students [73]; further, besides correlation analysis, Huang et al. operated a Least Absolute Shrinkage and Selection Operator (LASSO) linear regression model to infer the causal relationship between mental health disorders and location semantics [4].

Though items above could summarize most of the D\&Is issues in this step in Personal Sensing and Crowd Sensing paradigms, 
there are also some side D\&Is issues for some problems that may exist in the mobile sensing data [290]. For example, ideally, the input of the data analysis algorithm is continuous and sufficient, while in mHealth Sensing contexts, the data streams collected may be sparse and biased due to some technical issues (e.g., operating system's restrictions on software running in the background) and varying users' usage behaviors (e.g., forgetting to wear the device or run the app); thus overcoming the insufficiency of data and effective modeling is an urgent problem to be solved [17], [291]. Additionally, similar side problems include how to analyze and understand the relationship between the complex dynamics of the health and multimodal factors [292], and how to integrate medical knowledge into algorithms pervasively and effectively [293], [294].

\section{Future Directions}

In this work, we reviewed a number of mHealth sensing apps, including applications and systems of personal sensing and crowd sensing for personalized medicine and population health respectively. We proposed two taxonomy systems for mHealth sensing systems from the perspectives of "Sensing Objectives" and "Sensing Paradigms", and here summarize the two taxonomy systems in Table III.

It is obvious that mHealth Sensing apps in both Personal Sensing and Crowd Sensing paradigms will continue to be promising research topics to solve both Personalized Medicine and Population Health problems, where some research problems such as sensing tasks, incentives, privacy, data quality, sensing schemes, data analysis methods are still not well addressed in mHealth Sensing life cycle. Based on the proposed taxonomy systems and identified gaps, we foresee the following research directions in future works.

Promoting both Personal and Population Health Benefits \& Well-Beings: Most of existing mHealth Sensing works solely focus on either individual user's personal health benefit (in Personal Sensing) or population health benefit (in Crowd Sensing) [17], [56], [211], [231]. While the two paradigms have their own advantages and disadvantages (e.g., Crowd Sensing pursues to discover population knowledge, which may conflict with providing personal health benefit to participants as incentives; Personal Sensing pays main attention to providing service for personal health benefit, ignoring that the information of a group of individuals can be leveraged for population research), the organizer could consider combining both of them to make full use of service capacity and collected information of mHealth Sensing apps/systems.

Collecting Health-related Data with High Fidelity: Ensuring data quality is crucial in mHealth Sensing contexts, especially the data fidelity in Crowd Sensing practices [209], [295]. Building health-related datasets costs enormous participants, monetary incentive, and monitoring devices in long terms, where biased/error data can lead to huge cost wastes and false conclusions which harms human well-being [296], [297]. However, existing methods are mainly in repeated validation or trust-based manners, which cause extra overhead or are useless in health-related data collection contexts [275]. To this end, there is obviously an urgent need to propose valid data quality ensuring approaches in the loops of data collection.

Insightful Knowledge Discovery from a Specific Group of Individuals: On the one hand, data analysis in Personal Sensing can be further improved in timeliness, adaptiveness, and personalization. For example, advances in machine learning algorithms help consider and incorporate user's long-term historical status and clinical medical knowledge into health status modeling to achieve the goals above [282], [298]. On the other hand, thanks to the scale of massive group data and the variety in patterns that individuals exhibit when they gather into groups, Crowd Sensing organizers could discover much collective knowledge by considering the characteristics such as behavioral patterns, pathological symptoms, age groups, or other settings, of unique groups of people [299].

Overcoming Data Limitations in mHealth Sensing: One of the focal points of data analysis in mHealth Sensing is to design valid algorithms to handle non-continuous, multimodal, and dynamic mHealth data [300]. Specifically, in mHealth Sensing practice, the data is mainly collected by mobile apps, which is affected by the usage of the users and sensors' working mode [301], causing the data partially missing in the time series and out of synchronization between sensors; also, the complex activities of individuals also make the data have strong dynamics and diversification [302], which is also a test of the analysis model. Thus it is also challenging to design algorithms to overcome the limitations in mHealth data.

Least Privilege Sensing for Privacy-Preserving mHealth: Privacy is yet another crucial factor for both mobile computing and health management and, thus no doubt, for mHealth [214], [215] as well. To secure the personal health data from potential leakages, encryption techniques [303], [304] have been frequently used and optimized for mHealth data management. In addition to data security, privacy protection that controls the access of mobile Apps to some critical information [305], [306] is also required to scale-up mHealth in societies. In this way, mobile developers frequently need to design and develop the apps with verification that minimizes data access privileges subject to the actual needs. Thus, a unified and integrated approach, combining the data security and privacy controls subject to principle of least privilege [307]-[309] for mHealth sensing, might be a promising direction for future research.

Ethical Issues in Human-Subject Studies: After-all, the research on mHealth sensing is huamn-subject studies, where human involve in-the-loop of experiments, data analysis, and information disclosures. Thus, certain ethical issues should be addressed and studied [310], [311]. Some of mHealth sensing apps, such as Sensus [2], already include protocol management and ethical review components in the system to monitor the whole life-cycles of mHealth crowd sensing. In the future direction, advanced protocol management, ethical review, and even prescription management [176] techniques should be studied, especially for commercially-used mHealth apps.

\section{LIMITATIONS AND CONCLUSIONS}

The mHealth Sensing is a practical approach in modern healthcare domain, which is being widely used for the objectives on either $(a)$ personalized medicine for individuals or $(b)$ 
TABLE III: Summary of two taxonomy systems for mHealth Personal Sensing and Crowd Sensing

\begin{tabular}{|c|l|l|l|l|}
\hline \multirow{2}{*}{} & \multicolumn{2}{|c|}{ Sensing Objectives (System I) } & \multicolumn{2}{c|}{ Sensing Paradigms and D\&Is (System II) } \\
\cline { 2 - 5 } & Personalized Medicine & \multicolumn{1}{|c|}{ Population Health } & Personal Sensing & Crowd Sensing \\
\hline $\begin{array}{c}\text { Sensing } \\
\text { Task Creation \& } \\
\text { Participation }\end{array}$ & $\begin{array}{l}\text { Service provision } \\
\text { for personalized } \\
\text { medicine seekers }\end{array}$ & $\begin{array}{l}\text { Recruitment provision } \\
\text { for population } \\
\text { health participants }\end{array}$ & $\begin{array}{l}\text { Improving user engagement } \\
\text { via clinical health services, } \\
\text { gamification and attractions }\end{array}$ & $\begin{array}{l}\text { Extra motivating participants' } \\
\text { performance via recruitment } \\
\text { with monetary incentives }\end{array}$ \\
\hline $\begin{array}{c}\text { Health } \\
\text { Surveillance \& } \\
\text { Data Collection }\end{array}$ & $\begin{array}{l}\text { More focusing on data } \\
\text { precision, longitudinal } \\
\text { coverage, and resources } \\
\text { saving }\end{array}$ & $\begin{array}{l}\text { More focusing on data } \\
\text { fidelity, population } \\
\text { coverage, and privacy } \\
\text { and security }\end{array}$ & $\begin{array}{l}\text { Using granularity-oriented } \\
\text { sensing schemes }\end{array}$ & $\begin{array}{l}\text { Using coverage-oriented } \\
\text { sensing schemes with trails, } \\
\text { surveys, and evaluations } \\
\text { schemes }\end{array}$ \\
\hline $\begin{array}{c}\text { Data Analysis \& } \\
\text { Knowledge } \\
\text { Discovery }\end{array}$ & $\begin{array}{l}\text { Improving mHealth } \\
\text { accuracy in risk } \\
\text { prediction and precision } \\
\text { in predictive interventions }\end{array}$ & $\begin{array}{l}\text { Pursuing depth and } \\
\text { coverage of health } \\
\text { monitoring in communities } \\
\text { and statistical power } \\
\text { of mHealth approaches }\end{array}$ & $\begin{array}{l}\text { Leveraging health outcomes } \\
\text { and progressions predictions, } \\
\text { and health status recognition } \\
\text { and interventions }\end{array}$ & $\begin{array}{l}\text { Leveraging population health } \\
\text { assessment and health } \\
\text { determinants discovery }\end{array}$ \\
\hline
\end{tabular}

public health for populations. In this work, we reviewed and summarized mHealth sensing Apps and systems that deployed over smartphones and commodity ubiquitous devices. Though we have tried our best to cover the important works in this area and related fields, this survey is still with several limitations. For example, this work did not include professional medical systems for medicare/rehabilitation/assisted living purposes, such as medical sensors [150]-[154], [312], Medical IoTs/CPSs [155]-[177], and medical robots [178]-[183]. Furthermore, there have been a number of great works surveying or reviewing this area and related fields [1], [15], [38], [92], [139], [155], [210], [214], [216], [220], [224], [252], [264], [275], [311], [313]-[318], while we have not compared our taxonomy systems with these works.

To systematically summarize the existing works and identify the potential directions in this emerging research domain, this work actually presents two novel taxonomy systems from two major perspectives (i.e., sensing objectives and sensing paradigms and Designs \& Implementations (D\&Is)) that can specify and classify apps/systems from steps in the life-cycle of mHealth Sensing: (1) Sensing Task Creation \& Participation, (2) Health Surveillance \& Data Collection, and (3) Data Analysis \& Knowledge Discovery. Through discussing the realworld Mobile Sensing apps/systems in the proposed taxonomy systems, most of the research problems in mHealth Sensing can be formally classified, and several future research directions are pointed out, targeting to provide structural knowledge and insightful ideas and guidance for researchers in the related field.

\section{REFERENCES}

[1] N. D. Lane, E. Miluzzo, H. Lu, D. Peebles, T. Choudhury, and A. T. Campbell, "A survey of mobile phone sensing," IEEE Communications magazine, vol. 48, no. 9, pp. 140-150, 2010.

[2] H. Xiong, Y. Huang, L. E. Barnes, and M. S. Gerber, "Sensus: a crossplatform, general-purpose system for mobile crowdsensing in humansubject studies," in Proceedings of the 2016 ACM international joint conference on pervasive and ubiquitous computing, 2016, pp. 415-426.
[3] I. Perez-Pozuelo, D. Spathis, E. A. Clifton, and C. Mascolo, "Wearables, smartphones, and artificial intelligence for digital phenotyping and health," in Digital Health. Elsevier, 2021, pp. 33-54.

[4] Y. Huang, H. Xiong, K. Leach, Y. Zhang, P. Chow, K. Fua, B. A. Teachman, and L. E. Barnes, "Assessing social anxiety using gps trajectories and point-of-interest data," in Proceedings of the 2016 ACM International Joint Conference on Pervasive and Ubiquitous Computing, 2016, pp. 898-903.

[5] J. C. Sieverdes, F. Treiber, C. Jenkins, and K. Hermayer, "Improving diabetes management with mobile health technology," The American journal of the medical sciences, vol. 345, no. 4, pp. 289-295, 2013.

[6] T. Choudhury, G. Borriello, S. Consolvo, D. Haehnel, B. Harrison, B. Hemingway, J. Hightower, P. Pedja, K. Koscher, A. LaMarca et al., "The mobile sensing platform: An embedded activity recognition system," IEEE Pervasive Computing, vol. 7, no. 2, pp. 32-41, 2008.

[7] N. D. Lane, M. Mohammod, M. Lin, X. Yang, H. Lu, S. Ali, A. Doryab, E. Berke, T. Choudhury, and A. Campbell, "Bewell: A smartphone application to monitor, model and promote wellbeing," in 5th international ICST conference on pervasive computing technologies for healthcare, vol. 10, 2011.

[8] M. Rabbi, S. Ali, T. Choudhury, and E. Berke, "Passive and in-situ assessment of mental and physical well-being using mobile sensors," in Proceedings of the 13th international conference on Ubiquitous computing, 2011, pp. 385-394.

[9] A. Sathyanarayana, S. Joty, L. Fernandez-Luque, F. Ofli, J. Srivastava, A. Elmagarmid, T. Arora, and S. Taheri, "Sleep quality prediction from wearable data using deep learning," JMIR mHealth and uHealth, vol. 4, no. 4, p. e125, 2016.

[10] G. C.-L. Hung, P.-C. Yang, C.-C. Chang, J.-H. Chiang, and Y.-Y. Chen, "Predicting negative emotions based on mobile phone usage patterns: an exploratory study," JMIR research protocols, vol. 5, no. 3, p. e160, 2016.

[11] S. Servia-Rodríguez, K. K. Rachuri, C. Mascolo, P. J. Rentfrow, N. Lathia, and G. M. Sandstrom, "Mobile sensing at the service of mental well-being: a large-scale longitudinal study," in Proceedings of the 26th International Conference on World Wide Web, 2017, pp. 103112.

[12] S. R. Steinhubl, E. D. Muse, and E. J. Topol, "Can mobile health technologies transform health care?" Jama, vol. 310, no. 22, pp. 23952396, 2013.

[13] S. Mukhopadhyay and O. A. Postolache, "Pervasive and mobile sensing and computing for healthcare," Technolo. Soc. Issues, 2014.

[14] U. Varshney, "Mobile health: Four emerging themes of research," Decision Support Systems, vol. 66, pp. 20-35, 2014.

[15] B. M. Silva, J. J. Rodrigues, I. de la Torre Díez, M. López-Coronado, and K. Saleem, "Mobile-health: A review of current state in 2015," Journal of biomedical informatics, vol. 56, pp. 265-272, 2015.

[16] R. Pryss, "Mobile crowdsensing in healthcare scenarios: taxonomy, 
conceptual pillars, smart mobile crowdsensing services," in Digital Phenotyping and Mobile Sensing. Springer, 2019, pp. 221-234.

[17] M. Boukhechba, A. N. Baglione, and L. E. Barnes, "Leveraging mobile sensing and machine learning for personalized mental health care," Ergonomics in Design, vol. 28, no. 4, pp. 18-23, 2020.

[18] Y.-T. Park, "Emerging new era of mobile health technologies," Healthcare informatics research, vol. 22, no. 4, p. 253, 2016.

[19] S. R. Steinhubl, E. D. Muse, and E. J. Topol, "The emerging field of mobile health," Science translational medicine, vol. 7, no. 283, pp. 283rv3-283rv3, 2015.

[20] L. Huang, Y. Xu, X. Chen, H. Li, and Y. Wu, "Design and implementation of location based mobile health system," in 2012 Fourth International Conference on Computational and Information Sciences. IEEE, 2012, pp. 919-922.

[21] D. Kelly and B. Caulfield, "An investigation into non-invasive physical activity recognition using smartphones," in 2012 Annual International Conference of the IEEE Engineering in Medicine and Biology Society. IEEE, 2012, pp. 3340-3343.

[22] S. H. Almotiri, M. A. Khan, and M. A. Alghamdi, "Mobile health (mhealth) system in the context of iot," in 2016 IEEE 4th international conference on future internet of things and cloud workshops (FiCloudW). IEEE, 2016, pp. 39-42.

[23] A. Lorenz and R. Oppermann, "Mobile health monitoring for the elderly: Designing for diversity," Pervasive and Mobile computing, vol. 5, no. 5, pp. 478-495, 2009.

[24] P. R. Sama, Z. J. Eapen, K. P. Weinfurt, B. R. Shah, and K. A. Schulman, "An evaluation of mobile health application tools," JMIR mHealth and uHealth, vol. 2, no. 2, p. e19, 2014.

[25] A. of Medical Sciences, "Stratified, personalised or p4 medicine: a new direction for placing the patient at the centre of healthcare and health education," 2015.

[26] A. Pokorska-Bocci, A. Stewart, G. S. Sagoo, A. Hall, M. Kroese, and H. Burton, "'personalized medicine': what's in a name?" Personalized Medicine, vol. 11, no. 2, pp. 197-210, 2014.

[27] O. Amft, J. Favela, S. S. Intille, M. Musolesi, and V. Kostakos, "Personalized pervasive health." IEEE Pervasive Comput., vol. 19, no. 3, pp. $11-13,2020$

[28] D. A. DeWalt, N. D. Berkman, S. Sheridan, K. N. Lohr, and M. P. Pignone, "Literacy and health outcomes," Journal of general internal medicine, vol. 19, no. 12, pp. 1228-1239, 2004.

[29] D. Kindig and G. Stoddart, "What is population health?" American journal of public health, vol. 93, no. 3, pp. 380-383, 2003.

[30] D. Callahan, "The who definition of 'health'," Hastings Center Studies, pp. 77-87, 1973.

[31] S. Boccia, "Why is personalized medicine relevant to public health?" 2014.

[32] J. L. Mega, M. S. Sabatine, and E. M. Antman, "Population and personalized medicine in the modern era," Jama, vol. 312, no. 19, pp. 1969-1970, 2014.

[33] N. Evangelatos, K. Satyamoorthy, and A. Brand, "Personalized health in a public health perspective," 2018.

[34] M. Lapinski, M. Feldmeier, and J. A. Paradiso, "Wearable wireless sensing for sports and ubiquitous interactivity," in SENSORS, 2011 IEEE. IEEE, 2011, pp. 1425-1428.

[35] J. Zhang, Y. Cao, M. Qiao, L. Ai, K. Sun, Q. Mi, S. Zang, Y. Zuo, $\mathrm{X}$. Yuan, and Q. Wang, "Human motion monitoring in sports using wearable graphene-coated fiber sensors," Sensors and Actuators A: Physical, vol. 274, pp. 132-140, 2018.

[36] S. Groth and Y. Krahn, "Sensing athletes: Sensory dimensions of recreational endurance sports," Journal of Ethnology and Folkloristics, vol. 11, no. 2, pp. 3-23, 2017.

[37] A. Korotin, N. Khromov, A. Stepanov, A. Lange, E. Burnaev, and A. Somov, "Towards understanding of esports athletes' potentialities: The sensing system for data collection and analysis," in 2019 IEEE SmartWorld, Ubiquitous Intelligence \& Computing, Advanced \& Trusted Computing, Scalable Computing \& Communications, Cloud \& Big Data Computing, Internet of People and Smart City Innovation (SmartWorld/SCALCOM/UIC/ATC/CBDCom/IOP/SCI). IEEE, 2019, pp. 1804-1810.

[38] X. Zhang, Z. Yang, W. Sun, Y. Liu, S. Tang, K. Xing, and X. Mao, "Incentives for mobile crowd sensing: A survey," IEEE Communications Surveys \& Tutorials, vol. 18, no. 1, pp. 54-67, 2015.

[39] B. Wagner III, E. Liu, S. D. Shaw, G. Iakovlev, L. Zhou, C. Harrington, G. Abowd, C. Yoon, S. Kumar, S. Murphy et al., "e wrapper: operationalizing engagement strategies in mhealth," in Proceedings of the 2017 ACM International Joint Conference on Pervasive and Ubiquitous
Computing and Proceedings of the 2017 ACM International Symposium on Wearable Computers, 2017, pp. 790-798.

[40] J.-H. Huh, "Big data analysis for personalized health activities: machine learning processing for automatic keyword extraction approach," Symmetry, vol. 10, no. 4, p. 93, 2018.

[41] J. Liu, H. Cao, Q. Li, F. Cai, X. Du, and M. Guizani, "A largescale concurrent data anonymous batch verification scheme for mobile healthcare crowd sensing," IEEE Internet of things Journal, vol. 6, no. 2, pp. 1321-1330, 2018.

[42] D. Zhang, L. Wang, H. Xiong, and B. Guo, " 4 w1h in mobile crowd sensing," IEEE Communications Magazine, vol. 52, no. 8, pp. 42-48, 2014.

[43] T. Hao, G. Xing, and G. Zhou, "isleep: Unobtrusive sleep quality monitoring using smartphones," in Proceedings of the 11th ACM Conference on Embedded Networked Sensor Systems, 2013, pp. 1-14.

[44] S. Abdullah and T. Choudhury, "Sensing technologies for monitoring serious mental illnesses," IEEE MultiMedia, vol. 25, no. 1, pp. 61-75, 2018.

[45] F. Wahle, T. Kowatsch, E. Fleisch, M. Rufer, and S. Weidt, "Mobile sensing and support for people with depression: a pilot trial in the wild," JMIR mHealth and uHealth, vol. 4, no. 3, p. e111, 2016.

[46] S. Leao and P. Izadpahani, "Factors motivating citizen engagement in mobile sensing: Insights from a survey of non-participants," Journal of Urban Technology, vol. 23, no. 4, pp. 85-103, 2016.

[47] H. Xiong, D. Zhang, Z. Guo, G. Chen, and L. E. Barnes, "Near-optimal incentive allocation for piggyback crowdsensing," IEEE Communications Magazine, vol. 55, no. 6, pp. 120-125, 2017.

[48] L. G. Jaimes and R. Steele, "Incentivization for health crowdsensing," in 2017 IEEE 15th Intl Conf on Dependable, Autonomic and Secure Computing, 15th Intl Conf on Pervasive Intelligence and Computing, 3rd Intl Conf on Big Data Intelligence and Computing and Cyber Science and Technology Congress (DASC/PiCom/DataCom/CyberSciTech). IEEE, 2017, pp. 139-146.

[49] S. Saeb, M. Zhang, C. J. Karr, S. M. Schueller, M. E. Corden, K. P. Kording, and D. C. Mohr, "Mobile phone sensor correlates of depressive symptom severity in daily-life behavior: an exploratory study," Journal of medical Internet research, vol. 17, no. 7, p. e175, 2015.

[50] L. Wang, D. Zhang, and H. Xiong, "effsense: Energy-efficient and costeffective data uploading in mobile crowdsensing," in Proceedings of the 2013 ACM conference on Pervasive and ubiquitous computing adjunct publication, 2013, pp. 1075-1086.

[51] H. Xiong, D. Zhang, L. Wang, and H. Chaouchi, "Emc 3: Energyefficient data transfer in mobile crowdsensing under full coverage constraint," IEEE Transactions on Mobile Computing, vol. 14, no. 7, pp. 1355-1368, 2014.

[52] M. Rabbi, A. Pfammatter, M. Zhang, B. Spring, and T. Choudhury, "Automated personalized feedback for physical activity and dietary behavior change with mobile phones: a randomized controlled trial on adults," JMIR mHealth and uHealth, vol. 3, no. 2, p. e42, 2015.

[53] G. Agapito, B. Calabrese, P. H. Guzzi, M. Cannataro, M. Simeoni, I. Caré, T. Lamprinoudi, G. Fuiano, and A. Pujia, "Dietos: A recommender system for adaptive diet monitoring and personalized food suggestion," in 2016 IEEE 12th International Conference on Wireless and Mobile Computing, Networking and Communications (WiMob). IEEE, 2016, pp. 1-8.

[54] D. Chen, J. Wang, W. Ruan, Q. Ni, and S. Helal, "Enabling cost-effective population health monitoring by exploiting spatiotemporal correlation: An empirical study," ACM Transactions on Computing for Healthcare, vol. 2, no. 2, pp. 1-19, 2021.

[55] S. Adibi, Mobile health: a technology road map. Springer, 2015, vol. 5.

[56] D. C. Mohr, M. Zhang, and S. M. Schueller, "Personal sensing: understanding mental health using ubiquitous sensors and machine learning," Annual review of clinical psychology, vol. 13, pp. 23-47, 2017.

[57] R. K. Ganti, F. Ye, and H. Lei, "Mobile crowdsensing: current state and future challenges," IEEE communications Magazine, vol. 49, no. 11, pp. 32-39, 2011.

[58] J. Zhu, P. Wu, X. Wang, and J. Zhang, "Sensec: Mobile security through passive sensing," in 2013 International Conference on Computing, Networking and Communications (ICNC). IEEE, 2013, pp. 1128-1133.

[59] N. B. Belloc and L. Breslow, "Relationship of physical health status and health practices," Preventive medicine, vol. 1, no. 3, pp. 409-421, 1972.

[60] M. P. Manary, W. Boulding, R. Staelin, and S. W. Glickman, "The patient experience and health outcomes." The New England journal of medicine, 2013.

[61] M. Marmot and R. Wilkinson, Social determinants of health. Oup Oxford, 2005. 
[62] W. H. Organization et al., "Mental health: strengthening our response," 2010.

[63] N. S. Jacobson, L. J. Roberts, S. B. Berns, and J. B. McGlinchey, "Methods for defining and determining the clinical significance of treatment effects: description, application, and alternatives." Journal of consulting and clinical psychology, vol. 67, no. 3, p. 300, 1999.

[64] M. C. Weinstein, L. B. Russell, M. R. Gold, J. E. Siegel et al., Costeffectiveness in health and medicine. Oxford university press, 1996.

[65] P. G. Smith, R. H. Morrow, and D. A. Ross, "Types of intervention and their development," in Field Trials of Health Interventions: A Toolbox. 3rd edition. OUP Oxford, 2015.

[66] L. M. Babrak, J. Menetski, M. Rebhan, G. Nisato, M. Zinggeler, N. Brasier, K. Baerenfaller, T. Brenzikofer, L. Baltzer, C. Vogler et al., "Traditional and digital biomarkers: two worlds apart?" Digital biomarkers, vol. 3, no. 2, pp. 92-102, 2019.

[67] R. S. McIntyre, Y. Lee, C. Rong, J. D. Rosenblat, E. Brietzke, Z. Pan, C. Park, M. Subramaniapillai, R.-M. Ragguett, R. B. Mansur et al., "Ecological momentary assessment of depressive symptoms using the mind. me application: Convergence with the patient health questionnaire-9 (phq-9)," Journal of Psychiatric Research, vol. 135, pp. 311-317, 2021

[68] M. N. Burns, M. Begale, J. Duffecy, D. Gergle, C. J. Karr, E. Giangrande, and D. C. Mohr, "Harnessing context sensing to develop a mobile intervention for depression," Journal of medical Internet research, vol. 13, no. 3, p. e55, 2011

[69] E. Anthes, "Mental health: there's an app for that," Nature News, vol. 532 , no. 7597 , p. 20,2016

[70] J. Asselbergs, J. Ruwaard, M. Ejdys, N. Schrader, M. Sijbrandij, and H. Riper, "Mobile phone-based unobtrusive ecological momentary assessment of day-to-day mood: an explorative study," Journal of medical Internet research, vol. 18, no. 3, p. e72, 2016.

[71] R. Kraft, W. Schlee, M. Stach, M. Reichert, B. Langguth, H. Baumeister, T. Probst, R. Hannemann, and R. Pryss, "Combining mobile crowdsensing and ecological momentary assessments in the healthcare domain," Frontiers in neuroscience, vol. 14, p. 164, 2020.

[72] P. I. Chow, K. Fua, Y. Huang, W. Bonelli, H. Xiong, L. E. Barnes, and B. A. Teachman, "Using mobile sensing to test clinical models of depression, social anxiety, state affect, and social isolation among college students," Journal of medical Internet research, vol. 19, no. 3, p. e62, 2017.

[73] M. Boukhechba, Y. Huang, P. Chow, K. Fua, B. A. Teachman, and L. E. Barnes, "Monitoring social anxiety from mobility and communication patterns," in Proceedings of the 2017 ACM International Joint Conference on Pervasive and Ubiquitous Computing and Proceedings of the 2017 ACM International Symposium on Wearable Computers, 2017, pp. 749-753.

[74] R. Wang, M. S. Aung, S. Abdullah, R. Brian, A. T. Campbell, T. Choudhury, M. Hauser, J. Kane, M. Merrill, E. A. Scherer et al., "Crosscheck: toward passive sensing and detection of mental health changes in people with schizophrenia," in Proceedings of the 2016 ACM International Joint Conference on Pervasive and Ubiquitous Computing, 2016, pp. 886-897.

[75] J.-K. Min, A. Doryab, J. Wiese, S. Amini, J. Zimmerman, and J. I. Hong, "Toss'n'turn: smartphone as sleep and sleep quality detector," in Proceedings of the SIGCHI conference on human factors in computing systems, 2014, pp. 477-486.

[76] W. Gu, Z. Yang, L. Shangguan, W. Sun, K. Jin, and Y. Liu, "Intelligent sleep stage mining service with smartphones," 2014.

[77] P. Sharmila, V. Schroderus, E. Lagerspetz, and E. Peltonen, "Towards understanding smartphone usage and sleep with a crowdsensing approach," in Adjunct Proceedings of the 2020 ACM International Joint Conference on Pervasive and Ubiquitous Computing and Proceedings of the 2020 ACM International Symposium on Wearable Computers, 2020, pp. 700-703.

[78] S. Abdullah, M. Matthews, E. L. Murnane, G. Gay, and T. Choudhury, "Towards circadian computing: "early to bed and early to rise" makes some of us unhealthy and sleep deprived," in Proceedings of the 2014 ACM international joint conference on pervasive and ubiquitous computing, 2014, pp. 673-684.

[79] M. A. Al-Taee, W. Al-Nuaimy, A. Al-Ataby, Z. J. Muhsin, and S. N. Abood, "Mobile health platform for diabetes management based on the internet-of-things," in 2015 IEEE Jordan Conference on Applied Electrical Engineering and Computing Technologies (AEECT). IEEE, 2015, pp. 1-5.

[80] G. Cappon, G. Acciaroli, M. Vettoretti, A. Facchinetti, and G. Sparacino, "Wearable continuous glucose monitoring sensors: a revolution in diabetes treatment," Electronics, vol. 6, no. 3, p. 65, 2017.
[81] S. Puhr, M. Derdzinski, J. B. Welsh, A. S. Parker, T. Walker, and D. A. Price, "Real-world hypoglycemia avoidance with a continuous glucose monitoring system's predictive low glucose alert," Diabetes technology \& therapeutics, vol. 21, no. 4, pp. 155-158, 2019.

[82] M. N. K. Boulos and K. Koh, "Smart city lifestyle sensing, big data, geo-analytics and intelligence for smarter public health decision-making in overweight, obesity and type 2 diabetes prevention: the research we should be doing," 2021.

[83] R. Basatneh, B. Najafi, and D. G. Armstrong, "Health sensors, smart home devices, and the internet of medical things: an opportunity for dramatic improvement in care for the lower extremity complications of diabetes," Journal of diabetes science and technology, vol. 12, no. 3, pp. 577-586, 2018.

[84] A. F. Otoom, E. E. Abdallah, Y. Kilani, A. Kefaye, and M. Ashour, "Effective diagnosis and monitoring of heart disease," International Journal of Software Engineering and Its Applications, vol. 9, no. 1, pp. 143-156, 2015.

[85] A. Triantafyllidis, C. Velardo, T. Chantler, S. A. Shah, C. Paton, R. Khorshidi, L. Tarassenko, K. Rahimi, S.-H. Investigators et al., "A personalised mobile-based home monitoring system for heart failure: the support-hf study," International journal of medical informatics, vol. 84, no. 10 , pp. 743-753, 2015.

[86] N. S. Ali, Z. A. Alkaream Alyasseri, and A. Abdulmohson, "Real-time heart pulse monitoring technique using wireless sensor network and mobile application." International Journal of Electrical \& Computer Engineering (2088-8708), vol. 8, no. 6, 2018.

[87] R. J. Czosek, J. Anderson, P. R. Khoury, T. K. Knilans, D. S. Spar, and B. S. Marino, "Utility of ambulatory monitoring in patients with congenital heart disease," The American journal of cardiology, vol. 111, no. 5, pp. 723-730, 2013.

[88] F. Koehler, K. Koehler, O. Deckwart, S. Prescher, K. Wegscheider, B.A. Kirwan, S. Winkler, E. Vettorazzi, L. Bruch, M. Oeff et al., "Efficacy of telemedical interventional management in patients with heart failure (tim-hf2): a randomised, controlled, parallel-group, unmasked trial," The Lancet, vol. 392, no. 10152, pp. 1047-1057, 2018.

[89] J. Evans, A. Papadopoulos, C. T. Silvers, N. Charness, W. R. Boot, L. Schlachta-Fairchild, C. Crump, M. Martinez, and C. B. Ent, "Remote health monitoring for older adults and those with heart failure: adherence and system usability," Telemedicine and e-Health, vol. 22, no. 6, pp. 480-488, 2016.

[90] T. Karhula, A.-L. Vuorinen, K. Rääpysjärvi, M. Pakanen, P. Itkonen, M. Tepponen, U.-M. Junno, T. Jokinen, M. van Gils, J. Lähteenmäki et al., "Telemonitoring and mobile phone-based health coaching among finnish diabetic and heart disease patients: randomized controlled trial," Journal of medical Internet research, vol. 17, no. 6, p. e153, 2015.

[91] H.-H. Hsu and C.-C. Chen, "Rfid-based human behavior modeling and anomaly detection for elderly care," Mobile Information Systems, vol. 6 , no. 4, pp. 341-354, 2010.

[92] F. Zhou, J. R. Jiao, S. Chen, and D. Zhang, "A case-driven ambient intelligence system for elderly in-home assistance applications," IEEE Transactions on Systems, Man, and Cybernetics, Part C (Applications and Reviews), vol. 41, no. 2, pp. 179-189, 2010.

[93] S. H. Marakkalage, S. Sarica, B. P. L. Lau, S. K. Viswanath, T. Balasubramaniam, C. Yuen, B. Yuen, J. Luo, and R. Nayak, "Understanding the lifestyle of older population: Mobile crowdsensing approach," IEEE Transactions on Computational Social Systems, vol. 6, no. 1, pp. 82-95, 2018.

[94] Q. Lin, D. Zhang, X. Huang, H. Ni, and X. Zhou, "Detecting wandering behavior based on gps traces for elders with dementia," in 2012 12th International Conference on Control Automation Robotics \& Vision (ICARCV). IEEE, 2012, pp. 672-677.

[95] K. Du, D. Zhang, X. Zhou, M. Mokhtari, M. Hariz, and W. Qin, "Hycare: A hybrid context-aware reminding framework for elders with mild dementia," in International Conference On Smart homes and health Telematics. Springer, 2008, pp. 9-17.

[96] N. Hezarjaribi, S. Mazrouee, and H. Ghasemzadeh, "Speech2health: A mobile framework for monitoring dietary composition from spoken data," IEEE journal of biomedical and health informatics, vol. 22, no. 1, pp. $252-264,2017$.

[97] L. Meegahapola, S. Ruiz-Correa, and D. Gatica-Perez, "Alone or with others? understanding eating episodes of college students with mobile sensing," in 19th International Conference on Mobile and Ubiquitous Multimedia, 2020, pp. 162-166.

[98] W. Schlee, R. C. Pryss, T. Probst, J. Schobel, A. Bachmeier, M. Reichert, and B. Langguth, "Measuring the moment-to-moment variability of tinnitus: the trackyourtinnitus smart phone app," Frontiers in aging neuroscience, vol. 8, p. 294, 2016 
[99] R. Pryss, W. Schlee, B. Hoppenstedt, M. Reichert, M. Spiliopoulou, B. Langguth, M. Breitmayer, and T. Probst, "Applying machine learning to daily-life data from the trackyourtinnitus mobile health crowdsensing platform to predict the mobile operating system used with high accuracy: Longitudinal observational study," Journal of Medical Internet Research, vol. 22, no. 6, p. e15547, 2020.

[100] R. Pryss, M. Reichert, J. Herrmann, B. Langguth, and W. Schlee, "Mobile crowd sensing in clinical and psychological trials-a case study," in 2015 IEEE 28th International Symposium on Computer-Based Medical Systems. IEEE, 2015, pp. 23-24.

[101] M. Mehdi, D. Schwager, R. Pryss, W. Schlee, M. Reichert, and F. J. Hauck, "Towards automated smart mobile crowdsensing for tinnitus research," in 2019 IEEE 32nd International Symposium on ComputerBased Medical Systems (CBMS). IEEE, 2019, pp. 75-80.

[102] R. Pryss, W. Schlee, B. Langguth, and M. Reichert, "Mobile crowdsensing services for tinnitus assessment and patient feedback," in 2017 IEEE International Conference on AI \& Mobile Services (AIMS). IEEE, 2017, pp. 22-29.

[103] T. Probst, R. C. Pryss, B. Langguth, J. P. Rauschecker, J. Schobel, M. Reichert, M. Spiliopoulou, W. Schlee, and J. Zimmermann, "Does tinnitus depend on time-of-day? an ecological momentary assessment study with the "trackyourtinnitus" application," Frontiers in aging neuroscience, vol. 9, p. 253, 2017.

[104] A. S. R. S. Rao and J. A. Vazquez, "Identification of covid-19 can be quicker through artificial intelligence framework using a mobile phonebased survey when cities and towns are under quarantine." Infection Control and Hospital Epidemiology, vol. 41, no. 7, pp. 826-830, 2020.

[105] N. Sharma, P. Krishnan, R. Kumar, S. Ramoji, S. R. Chetupalli, P. K. Ghosh, S. Ganapathy et al., "Coswara-a database of breathing, cough, and voice sounds for covid-19 diagnosis," arXiv preprint arXiv:2005.10548, 2020.

[106] C. Brown, J. Chauhan, A. Grammenos, J. Han, A. Hasthanasombat, D. Spathis, T. Xia, P. Cicuta, and C. Mascolo, "Exploring automatic diagnosis of covid-19 from crowdsourced respiratory sound data," in Proceedings of the 26th ACM SIGKDD International Conference on Knowledge Discovery \& Data Mining, 2020, pp. 3474-3484.

[107] J. Han, C. Brown, J. Chauhan, A. Grammenos, A. Hasthanasombat, D. Spathis, T. Xia, P. Cicuta, and C. Mascolo, "Exploring automatic covid-19 diagnosis via voice and symptoms from crowdsourced data," in ICASSP 2021-2021 IEEE International Conference on Acoustics, Speech and Signal Processing (ICASSP). IEEE, 2021, pp. 8328-8332.

[108] C. Xiong, S. Hu, M. Yang, W. Luo, and L. Zhang, "Mobile device data reveal the dynamics in a positive relationship between human mobility and covid-19 infections." Proceedings of the National Academy of Sciences of the United States of America, vol. 117, no. 44, pp. $27087-$ 27 089, 2020.

[109] Y. Kang, S. Gao, Y. Liang, M. Li, J. Rao, and J. Kruse, "Multiscale dynamic human mobility flow dataset in the u.s. during the covid-19 epidemic," Scientific Data, vol. 7, no. 1, pp. 390-390, 2020.

[110] K. H. Grantz, H. R. Meredith, D. A. T. Cummings, C. J. E. Metcalf, B. T. Grenfell, J. R. Giles, S. Mehta, S. Solomon, A. Labrique, N. Kishore, C. O. Buckee, and A. Wesolowski, "The use of mobile phone data to inform analysis of covid-19 pandemic epidemiology," Nature Communications, vol. 11, no. 1, p. 4961, 2020.

[111] Q. Hao, L. Chen, F. Xu, and Y. Li, "Understanding the urban pandemic spreading of covid-19 with real world mobility data," in Proceedings of the 26th ACM SIGKDD International Conference on Knowledge Discovery \& Data Mining, 2020, pp. 3485-3492.

[112] H. Cho, D. Ippolito, and Y. W. Yu, "Contact tracing mobile apps for covid-19: Privacy considerations and related trade-offs." arXiv preprint arXiv:2003.11511, 2020

[113] M. Ienca and E. Vayena, "On the responsible use of digital data to tackle the covid-19 pandemic." Nature Medicine, vol. 26, no. 4, pp. 463-464, 2020.

[114] A. D. Carli, M. F. Franco, A. Gassmann, C. Killer, B. Rodrigues, E. J. Scheid, D. Schoenbaechler, and B. Stiller, "Wetrace - a privacypreserving mobile covid-19 tracing approach and application," arXiv preprint arXiv:2004.08812, 2020.

[115] Y. Xia and G. Lee, "How to return to normalcy: Fast and comprehensive contact tracing of covid-19 through proximity sensing using mobile devices," arXiv preprint arXiv:2004.12576, 2020.

[116] L. D. Domenico, G. Pullano, C. E. Sabbatini, P. Y. Boëlle, and V. Colizza, "Impact of lockdown on covid-19 epidemic in Île-de-france and possible exit strategies.” BMC Medicine, vol. 18, no. 1, pp. 1-13, 2020.

[117] M. Vinceti, T. Filippini, K. J. Rothman, F. Ferrari, A. Goffi, G. Maffeis, and N. Orsini, "Lockdown timing and efficacy in controlling covid-19 using mobile phone tracking." EClinicalMedicine, vol. 25, p. 100457 , 2020.

[118] J. Huang, H. Wang, H. Xiong, M. Fan, A. Zhuo, Y. Li, and D. Dou, "Quantifying the economic impact of covid-19 in mainland china using human mobility data," arXiv preprint arXiv:2005.03010, 2020.

[119] S. Gao, J. Rao, Y. Kang, Y. Liang, J. Kruse, D. Dopfer, A. K. Sethi, J. F. M. Reyes, B. S. Yandell, and J. A. Patz, "Association of mobile phone location data indications of travel and stay-at-home mandates with covid-19 infection rates in the us." JAMA Network Open, vol. 3, no. 9, 2020.

[120] Y. Zhou, R. Xu, D. Hu, Y. Yue, Q. Li, and J. Xia, "Effects of human mobility restrictions on the spread of covid-19 in shenzhen, china: a modelling study using mobile phone data," The Lancet. Digital health, vol. 2, no. 8, 2020 .

[121] J. Liu, X. Wang, H. Xiong, J. Huang, S. Huang, H. An, D. Dou, and $\mathrm{H}$. Wang, "An investigation of containment measures against the covid19 pandemic in mainland china," in 2020 IEEE International Conference on Big Data (Big Data), 2020, pp. 3204-3211.

[122] N. Gozzi, M. Tizzoni, M. Chinazzi, L. Ferres, A. Vespignani, and N. Perra, "Estimating the effect of social inequalities in the mitigation of covid-19 across communities in santiago de chile," medRxiv, 2020.

[123] H. Xiong, J. Liu, J. Huang, S. Huang, H. An, Q. Kang, Y. Li, D. Dou, and $\mathrm{H}$. Wang, "Understanding the collective responses of populations to the covid-19 pandemic in mainland china," medRxiv, 2020. [Online]. Available: https://www.medrxiv.org/content/early/2020/05/22/2020.04. 20.20068676

[124] J. Liu, T. Huang, H. Xiong, J. Huang, J. Zhou, H. Jiang, G. Yang, H. Wang, and D. Dou, "Analysis of collective response reveals that covid-19-related activities start from the end of 2019 in mainland china," Archives of Clinical and Biomedical Research, vol. 5, pp. 313-343, 2021.

[125] M. H. Aung, M. Matthews, and T. Choudhury, "Sensing behavioral symptoms of mental health and delivering personalized interventions using mobile technologies," Depression and anxiety, vol. 34, no. 7, pp. 603-609, 2017.

[126] N. Palmius, A. Tsanas, K. Saunders, A. C. Bilderbeck, J. R. Geddes, G. M. Goodwin, and M. De Vos, "Detecting bipolar depression from geographic location data," IEEE Transactions on Biomedical Engineering, vol. 64, no. 8, pp. 1761-1771, 2016.

[127] H. Ritchie and M. Roser, "Mental health," Our World in Data, 2018, https://ourworldindata.org/mental-health.

[128] J. Bronson and M. Berzofsky, "Indicators of mental health problems reported by prisoners and jail inmates, 2011-12," Bureau of Justice Statistics, pp. 1-16, 2017.

[129] F. Gravenhorst, A. Muaremi, J. Bardram, A. Grünerbl, O. Mayora, G. Wurzer, M. Frost, V. Osmani, B. Arnrich, P. Lukowicz et al., "Mobile phones as medical devices in mental disorder treatment: an overview," Personal and Ubiquitous Computing, vol. 19, no. 2, pp. 335-353, 2015.

[130] J. Costa, A. T. Adams, M. F. Jung, F. Guimbretière, and T. Choudhury, "Emotioncheck: A wearable device to regulate anxiety through false heart rate feedback," GetMobile: Mobile Computing and Communications, vol. 21, no. 2, pp. 22-25, 2017.

[131] E. Rey, A. Jain, S. Abdullah, T. Choudhury, and D. Erickson, "Personalized stress monitoring: a smartphone-enabled system for quantification of salivary cortisol," Personal and Ubiquitous Computing, vol. 22, no. 4, pp. 867-877, 2018 .

[132] V. W.-S. Tseng, N. Valliappan, V. Ramachandran, T. Choudhury, and V. Navalpakkam, "Digital biomarker of mental fatigue," NPJ digital medicine, vol. 4, no. 1, pp. 1-5, 2021.

[133] J. Torous, P. Staples, and J.-P. Onnela, "Realizing the potential of mobile mental health: new methods for new data in psychiatry," Current psychiatry reports, vol. 17, no. 8, pp. 1-7, 2015.

[134] W. Wang, S. Mirjafari, G. Harari, D. Ben-Zeev, R. Brian, T. Choudhury, M. Hauser, J. Kane, K. Masaba, S. Nepal et al., "Social sensing: assessing social functioning of patients living with schizophrenia using mobile phone sensing," in Proceedings of the 2020 CHI Conference on Human Factors in Computing Systems, 2020, pp. 1-15.

[135] I. Nahum-Shani, S. N. Smith, B. J. Spring, L. M. Collins, K. Witkiewitz, A. Tewari, and S. A. Murphy, "Just-in-time adaptive interventions (jitais) in mobile health: key components and design principles for ongoing health behavior support," Annals of Behavioral Medicine, vol. 52, no. 6, pp. 446-462, 2018.

[136] M. Menictas, M. Rabbi, P. Klasnja, and S. Murphy, "Artificial intelligence decision-making in mobile health," The Biochemist, vol. 41, no. 5, pp. 20-24, 2019. 
[137] M. Menictas, S. Tomkins, and S. Murphy, "Fast physical activity suggestions: Efficient hyperparameter learning in mobile health," arXiv preprint arXiv:2012.11646, 2020.

[138] T. J. VanderWeele, J. W. Jackson, and S. Li, "Causal inference and longitudinal data: a case study of religion and mental health," Social psychiatry and psychiatric epidemiology, vol. 51, no. 11, pp. 1457-1466, 2016.

[139] N. Surantha, G. P. Kusuma, and S. M. Isa, "Internet of things for sleep quality monitoring system: A survey," in 2016 11th International Conference on Knowledge, Information and Creativity Support Systems (KICSS). IEEE, 2016, pp. 1-6.

[140] M. De Zambotti, N. Cellini, A. Goldstone, I. M. Colrain, and F. C. Baker, "Wearable sleep technology in clinical and research settings," Medicine and science in sports and exercise, vol. 51, no. 7, p. 1538, 2019.

[141] D. Looney, V. Goverdovsky, I. Rosenzweig, M. J. Morrell, and D. P. Mandic, "Wearable in-ear encephalography sensor for monitoring sleep. preliminary observations from nap studies," Annals of the American Thoracic Society, vol. 13, no. 12, pp. 2229-2233, 2016.

[142] X. B. Pan, "Application of personal-oriented digital technology in preventing transmission of covid-19, china." Irish Journal of Medical Science, vol. 189, no. 4, pp. 1145-1146, 2020.

[143] N. Oliver, B. Lepri, H. Sterly, R. Lambiotte, S. Deletaille, M. D Nadai, E. Letouzé, A. A. Salah, R. Benjamins, C. Cattuto, V. Colizza, N. de Cordes, S. P. Fraiberger, T. Koebe, S. Lehmann, J. Murillo, A. Pentland, P. N. Pham, F. Pivetta, J. Saramäki, S. V. Scarpino, M. Tizzoni, S. Verhulst, and P. Vinck, "Mobile phone data for informing public health actions across the covid-19 pandemic life cycle." Science Advances, vol. 6, no. 23, 2020.

[144] D. Chamberlain, R. Kodgule, D. Ganelin, V. Miglani, and R. R. Fletcher, "Application of semi-supervised deep learning to lung sound analysis,' in 2016 38th Annual International Conference of the IEEE Engineering in Medicine and Biology Society (EMBC). IEEE, 2016, pp. 804-807.

[145] W. H. Organization et al., "Coronavirus disease (covid-19): How is it transmitted," 2020.

[146] N. N. DePhillipo, J. Chahla, M. Busler, and R. F. LaPrade, "Mobile phone gps data and prevalence of covid-19 infections: Quantifying parameters of social distancing in the u.s," The archives of bone and joint surgery, vol. 9, no. 2, pp. 217-223, 2021.

[147] (2020) Baidu qianxi. [Online]. Available: https://qianxi.baidu.com

[148] A. Arun, A. Gupta, S. Bhatka, S. Komatineni, and D. Bharadia, "Bluble, space-time social distancing to monitor the spread of covid-19: poster abstract," in Proceedings of the 18th Conference on Embedded Networked Sensor Systems, 2020, pp. 750-751.

[149] N. Kishore, M. Kiang, K. Engø-Monsen, N. Vembar, A. Schroeder, S. Balsari, and C. O. Buckee, "Measuring mobility to monitor travel and physical distancing interventions: a common framework for mobile phone data analysis," reponame:Expeditio Repositorio Institucional UJTL, 2020.

[150] L. R. Beck, B. M. Lobitz, and B. L. Wood, "Remote sensing and human health: new sensors and new opportunities." Emerging Infectious Diseases, vol. 6, no. 3, pp. 217-227, 2000.

[151] L. Y. Chen, B. C. K. Tee, A. L. Chortos, G. Schwartz, V. Tse, D. J Lipomi, H. S. P. Wong, M. V. McConnell, and Z. Bao, "Continuous wireless pressure monitoring and mapping with ultra-small passive sensors for health monitoring and critical care," Nature Communications, vol. 5, no. 1, p. 5028, 2014.

[152] B. S. Khakh and R. A. North, "P2x receptors as cell-surface atp sensors in health and disease," Nature, vol. 442, no. 7102, pp. 527-532, 2006.

[153] A. T. Güntner, S. Abegg, K. Königstein, P. A. Gerber, A. SchmidtTrucksäss, and S. E. Pratsinis, "Breath sensors for health monitoring," ACS Sensors, vol. 4, no. 2, pp. 268-280, 2019.

[154] Y. Gao, L. Yu, J. C. Yeo, and C. T. Lim, "Flexible hybrid sensors for health monitoring: Materials and mechanisms to render wearability." Advanced Materials, vol. 32, no. 15, p. 1902133, 2020.

[155] A. Gatouillat, Y. Badr, B. Massot, and E. Sejdic, "Internet of medica things: A review of recent contributions dealing with cyber-physical systems in medicine," IEEE Internet of Things Journal, vol. 5, no. 5, pp. 3810-3822, 2018.

[156] D. V. Dimitrov, "Medical internet of things and big data in healthcare," Healthcare Informatics Research, vol. 22, no. 3, pp. 156-163, 2016.

[157] M. Haghi, K. Thurow, and R. Stoll, "Wearable devices in medical internet of things: Scientific research and commercially available devices." Healthcare Informatics Research, vol. 23, no. 1, pp. 4-15, 2017.

[158] B. A. Hayani and H. Ilhan, "Image transmission over decode and forward based cooperative wireless multimedia sensor networks for rayleigh fading channels in medical internet of things (miot) for remote health-care and health communication monitoring," Journal of Medical Imaging and Health Informatics, vol. 10, no. 1, pp. 160-168, 2020.

[159] G. J. Joyia, R. M. Liaqat, A. Farooq, and S. Rehman, "Internet of medical things (iomt): Applications, benefits and future challenges in healthcare domain," Journal of Communication, vol. 12, pp. 240-247, 2017.

[160] M. Elhoseny, G.-B. Bian, S. Lakshmanaprabu, K. Shankar, A. K. Singh, and W. Wu, "Effective features to classify ovarian cancer data in internet of medical things," Computer Networks, vol. 159, pp. 147-156, 2019.

[161] A. Pazienza, R. Anglani, G. Mallardi, C. Fasciano, P. Noviello, C. Tatulli, and F. Vitulano, "Adaptive critical care intervention in the internet of medical things," in 2020 IEEE Conference on Evolving and Adaptive Intelligent Systems (EAIS), 2020, pp. 1-8.

[162] A. Limaye and T. Adegbija, "Hermit: A benchmark suite for the internet of medical things," IEEE Internet of Things Journal, vol. 5, no. 5, pp. 4212-4222, 2018.

[163] N. B. Gayathri, G. Thumbur, P. R. Kumar, M. Z. U. Rahman, P. V. Reddy, and A. Lay-Ekuakille, "Efficient and secure pairing-free certificateless aggregate signature scheme for healthcare wireless medical sensor networks," IEEE Internet of Things Journal, vol. 6, no. 5, pp. 9064-9075, 2019.

[164] R. Atat, L. Liu, J. Ashdown, M. J. Medley, J. D. Matyjas, and Y. Yi, "A physical layer security scheme for mobile health cyber-physical systems," IEEE Internet of Things Journal, vol. 5, no. 1, pp. 295-309, 2018.

[165] M. Haghi, S. Neubert, A. Geissler, H. Fleischer, N. Stoll, R. Stoll, and $\mathrm{K}$. Thurow, "A flexible and pervasive iot-based healthcare platform for physiological and environmental parameters monitoring," IEEE Internet of Things Journal, vol. 7, no. 6, pp. 5628-5647, 2020.

[166] M. A. Rahman and M. S. Hossain, "m-therapy: A multisensor framework for in-home therapy management: A social therapy of things perspective," IEEE Internet of Things Journal, vol. 5, no. 4, pp. 25482556, 2018.

[167] L. Jiang, L. Chen, T. Giannetsos, B. Luo, K. Liang, and J. Han, "Toward practical privacy-preserving processing over encrypted data in iot: An assistive healthcare use case," IEEE Internet of Things Journal, vol. 6, no. 6, pp. $10177-10190,2019$.

[168] S. A. Parah, J. A. Kaw, P. Bellavista, N. A. Loan, G. Bhat, K. Muhammad, and A. Victor, "Efficient security and authentication for edge-based internet of medical things," IEEE Internet of Things Journal, pp. 1-1, 2020.

[169] H. Peng, B. Yang, L. Li, and Y. Yang, "Secure and traceable image transmission scheme based on semitensor product compressed sensing in telemedicine system," IEEE Internet of Things Journal, vol. 7, no. 3, pp. 2432-2451, 2020.

[170] J. Liu, L. Wang, and Y. Yu, "Improved security of a pairing-free certificateless aggregate signature in healthcare wireless medical sensor networks," IEEE Internet of Things Journal, vol. 7, no. 6, pp. 52565266, 2020.

[171] R. Zhang, A. Nayak, S. Zhang, and J. Yu, "Energy-efficient sleep scheduling in wbans: From the perspective of minimum dominating set," IEEE Internet of Things Journal, vol. 6, no. 4, pp. 6237-6246, 2019.

[172] W. Meng, Y. Cai, L. T. Yang, and W.-Y. Chiu, "Hybrid emotion-aware monitoring system based on brainwaves for internet of medical things," IEEE Internet of Things Journal, no. 99, pp. 1-1, 2021.

[173] S. Amendola, R. Lodato, S. Manzari, C. Occhiuzzi, and G. Marrocco, "Rfid technology for iot-based personal healthcare in smart spaces," IEEE Internet of Things Journal, vol. 1, no. 2, pp. 144-152, 2014.

[174] B. Lin and S. J. Wu, "Covid-19 (coronavirus disease 2019): Opportunities and challenges for digital health and the internet of medical things in china." Omics A Journal of Integrative Biology, vol. 24, no. 5, pp. 231-232, 2020.

[175] P. Sundaravadivel, E. Kougianos, S. P. Mohanty, and M. K. Ganapathiraju, "Everything you wanted to know about smart health care: Evaluating the different technologies and components of the internet of things for better health," IEEE Consumer Electronics Magazine, vol. 7, no. 1, pp. 18-28, 2018.

[176] M. M. Hossain, S. M. R. Islam, F. Ali, K. S. Kwak, and R. Hasan, "An internet of things-based health prescription assistant and its security system design," Future Generation Computer Systems, vol. 82, pp. 422439, 2018.

[177] N. Zhu, T. Diethe, M. Camplani, L. Tao, A. Burrows, N. Twomey, D. Kaleshi, M. Mirmehdi, P. Flach, and I. Craddock, "Bridging e-health and the internet of things: The sphere project," IEEE Intelligent Systems, vol. 30, no. 4, pp. 39-46, 2015.

[178] M. A. Al-Taee, W. Al-Nuaimy, Z. J. Muhsin, and A. Al-Ataby, "Robot assistant in management of diabetes in children based on the internet 
of things," IEEE Internet of Things Journal, vol. 4, no. 2, pp. 437-445, 2017.

[179] R. Taylor and D. Stoianovici, "Medical robotics in computer-integrated surgery," international conference on robotics and automation, vol. 19, no. 5, pp. 765-781, 2003.

[180] R. J. Webster, J. S. Kim, N. J. Cowan, G. S. Chirikjian, and A. M. Okamura, "Nonholonomic modeling of needle steering," The International Journal of Robotics Research, vol. 25, no. 5, pp. 509-525, 2006.

[181] H. Wang, Z. Hou, H. Liu, X. Zheng, and X. Shi, "Lower limb rehabilitation medical robot used for paralytic patient," 2011.

[182] R. A. Beasley, "Medical robots: Current systems and research directions," Journal of Robotics, vol. 2012, no. 2012, pp. 1-14, 2012.

[183] T. Fukushima, W. Kokubo, T. Tsuboi, A. Miyamoto, K. Nagasaka, and K. Hirose, "Medical robot arm apparatus, medical robot arm control system, medical robot arm control method, and program," 2014.

[184] W. T. Riley, D. E. Rivera, A. A. Atienza, W. Nilsen, S. M. Allison, and R. Mermelstein, "Health behavior models in the age of mobile interventions: are our theories up to the task?" Translational behavioral medicine, vol. 1, no. 1, pp. 53-71, 2011.

[185] P. Klasnja, S. Consolvo, D. W. McDonald, J. A. Landay, and W. Pratt, "Using mobile \& personal sensing technologies to support health behavior change in everyday life: lessons learned," in AMIA Annual Symposium Proceedings, vol. 2009. American Medical Informatics Association, 2009, p. 338.

[186] S. Asimakopoulos, G. Asimakopoulos, and F. Spillers, "Motivation and user engagement in fitness tracking: Heuristics for mobile healthcare wearables," in Informatics, vol. 4, no. 1. Multidisciplinary Digital Publishing Institute, 2017, p. 5

[187] D. Spathis, S. Servia-Rodriguez, K. Farrahi, C. Mascolo, and J. Rentfrow, "Sequence multi-task learning to forecast mental wellbeing from sparse self-reported data," in Proceedings of the 25th ACM SIGKDD International Conference on Knowledge Discovery \& Data Mining, 2019, pp. 2886-2894.

[188] M. Boukhechba and L. E. Barnes, "Swear: Sensing using wearables. generalized human crowdsensing on smartwatches," in International Conference on Applied Human Factors and Ergonomics. Springer, 2020, pp. 510-516.

[189] M. T. Rashid and D. Wang, "Covidsens: a vision on reliable social sensing for covid-19," Artificial intelligence review, pp. 1-25, 2020

[190] D. Ben-Zeev, B. Buck, A. Chander, R. Brian, W. Wang, D. Atkins, C. J. Brenner, T. Cohen, A. Campbell, and J. Munson, "Mobile rdoc: Using smartphones to understand the relationship between auditory verbal hallucinations and need for care," Schizophrenia Bulletin Open, vol. 1, no. 1, p. sgaa060, 2020.

[191] W. Peng, S. Kanthawala, S. Yuan, and S. A. Hussain, "A qualitative study of user perceptions of mobile health apps," BMC public health, vol. 16, no. 1, pp. 1-11, 2016.

[192] R. Y. Wang and D. M. Strong, "Beyond accuracy: What data quality means to data consumers," Journal of management information systems, vol. 12, no. 4, pp. 5-33, 1996.

[193] N. A. Halpern, D. A. Goldman, K. S. Tan, and S. M. Pastores, "Trends in critical care beds and use among population groups and medicare and medicaid beneficiaries in the united states: 2000-2010," Critical care medicine, vol. 44, no. 8, p. 1490, 2016.

[194] A. S. Ahouandjinou, K. Assogba, and C. Motamed, "Smart and pervasive icu based-iot for improving intensive health care," in 2016 International Conference on Bio-engineering for Smart Technologies (BioSMART). IEEE, 2016, pp. 1-4.

[195] A. Davoudi, K. R. Malhotra, B. Shickel, S. Siegel, S. Williams, M. Ruppert, E. Bihorac, T. Ozrazgat-Baslanti, P. J. Tighe, A. Bihorac et al., "Intelligent icu for autonomous patient monitoring using pervasive sensing and deep learning," Scientific reports, vol. 9, no. 1, pp. 1-13, 2019.

[196] B. Roshanaei-Moghaddam, W. J. Katon, and J. Russo, "The longitudinal effects of depression on physical activity," General hospital psychiatry, vol. 31, no. 4, pp. 306-315, 2009.

[197] S. Fong, Y. Zhuang, S. Hu, W. Song, L. Liu, and L. A. Moutinho, "Longitudinal ambient mobile sensor monitoring for tcm-oriented healthcare assessments: Framework, challenges and applications," in Proceedings of the 26th International Conference on World Wide Web Companion, 2017, pp. 1087-1094.

[198] M. Kalanadhabhatta, T. Rahman, and D. Ganesan, "Effect of sleep and biobehavioral patterns on multidimensional cognitive performance: Longitudinal, in-the-wild study," Journal of Medical Internet Research, vol. 23 , no. 2, p. e23936, 2021.

[199] G. M. Harari, N. D. Lane, R. Wang, B. S. Crosier, A. T. Campbell, and S. D. Gosling, "Using smartphones to collect behavioral data in psychological science: Opportunities, practical considerations, and challenges," Perspectives on Psychological Science, vol. 11, no. 6, pp. 838-854, 2016.

[200] L. McNamara and E. Ngai, "Sadhealth: a personal mobile sensing system for seasonal health monitoring," IEEE systems journal, vol. 12, no. 1, pp. 30-40, 2016

[201] S. Majumder and M. J. Deen, "Smartphone sensors for health monitoring and diagnosis," Sensors, vol. 19, no. 9, p. 2164, 2019.

[202] R. K. Balan, Y. Lee, T. K. Wee, and A. Misra, "The challenge of continuous mobile context sensing," in 2014 Sixth International Conference on Communication Systems and Networks (COMSNETS). IEEE, 2014, pp. $1-8$.

[203] J. Wang, Y. Wang, D. Zhang, and S. Helal, "Energy saving techniques in mobile crowd sensing: Current state and future opportunities," IEEE Communications Magazine, vol. 56, no. 5, pp. 164-169, 2018.

[204] F. Ben Abdesslem, A. Phillips, and T. Henderson, "Less is more: energyefficient mobile sensing with senseless," in Proceedings of the 1st ACM workshop on Networking, systems, and applications for mobile handhelds, 2009, pp. 61-62.

[205] N. D. Lane and P. Georgiev, "Can deep learning revolutionize mobile sensing?" in Proceedings of the 16th International Workshop on Mobile Computing Systems and Applications, 2015, pp. 117-122.

[206] A. Anjomshoaa, F. Duarte, D. Rennings, T. J. Matarazzo, P. deSouza, and C. Ratti, "City scanner: Building and scheduling a mobile sensing platform for smart city services," IEEE Internet of things Journal, vol. 5 , no. 6, pp. 4567-4579, 2018.

[207] P. Gilbert, L. P. Cox, J. Jung, and D. Wetherall, "Toward trustworthy mobile sensing," in Proceedings of the Eleventh Workshop on Mobile Computing Systems \& Applications, 2010, pp. 31-36.

[208] M. Rahman, N. Ali, R. Bari, N. Saleheen, M. al'Absi, E. Ertin, A. Kennedy, K. L. Preston, and S. Kumar, "mdebugger: Assessing and diagnosing the fidelity and yield of mobile sensor data," in Mobile Health. Springer, 2017, pp. 121-143.

[209] M. M. Rahman, V. Nathan, E. Nemati, K. Vatanparvar, M. Ahmed, and J. Kuang, "Towards reliable data collection and annotation to extract pulmonary digital biomarkers using mobile sensors," in Proceedings of the 13th EAI International Conference on Pervasive Computing Technologies for Healthcare, 2019, pp. 179-188.

[210] A. Trifan, M. Oliveira, and J. L. Oliveira, "Passive sensing of health outcomes through smartphones: systematic review of current solutions and possible limitations," JMIR mHealth and uHealth, vol. 7, no. 8, p. e12649, 2019.

[211] Y. Zhang, F. Xu, T. Li, V. Kostakos, P. Hui, and Y. Li, "Passive health monitoring using large scale mobility data," Proceedings of the ACM on Interactive, Mobile, Wearable and Ubiquitous Technologies, vol. 5 , no. 1, pp. 1-23, 2021.

[212] J. Wiens and E. S. Shenoy, "Machine learning for healthcare: on the verge of a major shift in healthcare epidemiology," Clinical Infectious Diseases, vol. 66, no. 1, pp. 149-153, 2018.

[213] W. Edwards, H. Lindman, and L. J. Savage, "Bayesian statistical inference for psychological research." Psychological review, vol. 70, no. 3, p. $193,1963$.

[214] B. Martínez-Pérez, I. De La Torre-Díez, and M. López-Coronado, "Privacy and security in mobile health apps: a review and recommendations," Journal of medical systems, vol. 39, no. 1, pp. 1-8, 2015.

[215] A. Papageorgiou, M. Strigkos, E. Politou, E. Alepis, A. Solanas, and C. Patsakis, "Security and privacy analysis of mobile health applications: the alarming state of practice," IEEE Access, vol. 6, pp. 9390-9403, 2018.

[216] Y. Wang, Z. Yan, W. Feng, and S. Liu, "Privacy protection in mobile crowd sensing: a survey," World Wide Web, vol. 23, no. 1, pp. 421-452, 2020.

[217] D. He, S. Chan, and M. Guizani, "User privacy and data trustworthiness in mobile crowd sensing," IEEE Wireless Communications, vol. 22, no. 1, pp. 28-34, 2015.

[218] D. Wu, S. Si, S. Wu, and R. Wang, "Dynamic trust relationships aware data privacy protection in mobile crowd-sensing," IEEE Internet of Things Journal, vol. 5, no. 4, pp. 2958-2970, 2017.

[219] J. Ni, K. Zhang, X. Lin, Q. Xia, and X. S. Shen, "Privacy-preserving mobile crowdsensing for located-based applications," in 2017 IEEE International Conference on Communications (ICC). IEEE, 2017, pp. $1-6$.

[220] L. Pournajaf, L. Xiong, D. A. Garcia-Ulloa, and V. Sunderam, "A survey on privacy in mobile crowd sensing task management," Dept. Math. Comput. Sci., Emory Univ., Atlanta, GA, USA, Tech. Rep. TR-2014-002, 2014. 
[221] L. Ma, Q. Pei, Y. Qu, K. Fan, and X. Lai, "Decentralized privacypreserving reputation management for mobile crowdsensing," in International Conference on Security and Privacy in Communication Systems. Springer, 2019, pp. 532-548.

[222] P. Singh, R. S. Bali, N. Kumar, A. K. Das, A. Vinel, and L. T. Yang, "Secure healthcare data dissemination using vehicle relay networks," IEEE Internet of Things Journal, vol. 5, no. 5, pp. 3733-3746, 2018.

[223] M. Kumar and S. Chand, "A secure and efficient cloud-centric internetof-medical-things-enabled smart healthcare system with public verifiability," IEEE Internet of Things Journal, vol. 7, no. 10, pp. 10650 $10659,2020$.

[224] N. Jothi, W. Husain et al., "Data mining in healthcare-a review," Procedia computer science, vol. 72, pp. 306-313, 2015.

[225] I. Olaronke and O. Oluwaseun, "Big data in healthcare: Prospects, challenges and resolutions," in 2016 Future Technologies Conference (FTC). IEEE, 2016, pp. 1152-1157.

[226] C. S. Wood, M. R. Thomas, J. Budd, T. P. Mashamba-Thompson, K. Herbst, D. Pillay, R. W. Peeling, A. M. Johnson, R. A. McKendry, and M. M. Stevens, "Taking connected mobile-health diagnostics of infectious diseases to the field," Nature, vol. 566, no. 7745, pp. 467-474, 2019

[227] M. Boukhechba, P. Chow, K. Fua, B. A. Teachman, and L. E. Barnes, "Predicting social anxiety from global positioning system traces of college students: feasibility study," JMIR mental health, vol. 5, no. 3 , p. e10101, 2018.

[228] V. W.-S. Tseng, A. Sano, D. Ben-Zeev, R. Brian, A. T. Campbell, M. Hauser, J. M. Kane, E. A. Scherer, R. Wang, W. Wang et al., "Using behavioral rhythms and multi-task learning to predict fine-grained symptoms of schizophrenia," Scientific reports, vol. 10, no. 1, pp. 1-17, 2020

[229] E. K. Barrett, C. M. Fard, H. N. Katinas, C. V. Moens, L. E. Perry, B. E. Ruddy, S. D. Shah, I. S. Tucker, T. J. Wilson, M. Rucker et al., "Mobile sensing: Leveraging machine learning for efficient human behavior modeling," in 2020 Systems and Information Engineering Design Symposium (SIEDS). IEEE, 2020, pp. 1-7.

[230] C. Sedgley, "The responsibilities of being a physiotherapist," Tidy's Physiotherapy, p. 1, 2013

[231] M. S. Fragala, D. Shiffman, and C. E. Birse, "Population health screenings for the prevention of chronic disease progression," Am. J. Manag. Care, vol. 25, pp. 548-553, 2019.

[232] R. Wang, W. Wang, M. S. Aung, D. Ben-Zeev, R. Brian, A. T. Campbell, T. Choudhury, M. Hauser, J. Kane, E. A. Scherer et al., "Predicting symptom trajectories of schizophrenia using mobile sensing," Proceedings of the ACM on Interactive, Mobile, Wearable and Ubiquitous Technologies, vol. 1, no. 3, pp. 1-24, 2017.

[233] N. Bidargaddi, G. Schrader, P. Klasnja, J. Licinio, and S. Murphy, "Designing m-health interventions for precision mental health support," Translational psychiatry, vol. 10, no. 1, pp. 1-8, 2020

[234] H. Lei, A. Tewari, and S. A. Murphy, "An actor-critic contextual bandit algorithm for personalized mobile health interventions," arXiv preprint arXiv:1706.09090, 2017.

[235] D. G. Clayton, L. Bernardinelli, and C. Montomoli, "Spatial correlation in ecological analysis," International journal of epidemiology, vol. 22, no. 6, pp. 1193-1202, 1993.

[236] E. R. Seaquist, F. C. Goetz, S. Rich, and J. Barbosa, "Familial clustering of diabetic kidney disease," New England Journal of Medicine, vol. 320, no. 18 , pp. $1161-1165,1989$

[237] J. M. Cecilia, J.-C. Cano, E. Hernández-Orallo, C. T. Calafate, and P. Manzoni, "Mobile crowdsensing approaches to address the covid-19 pandemic in spain," IET Smart Cities, vol. 2, no. 2, pp. 58-63, 2020.

[238] E. S. Poole, "Hci and mobile health interventions: how human-computer interaction can contribute to successful mobile health interventions," Translational behavioral medicine, vol. 3, no. 4, pp. 402-405, 2013

[239] R. Schnall, M. Rojas, S. Bakken, W. Brown, A. Carballo-Dieguez, M. Carry, D. Gelaude, J. P. Mosley, and J. Travers, "A user-centered model for designing consumer mobile health (mhealth) applications (apps)," Journal of biomedical informatics, vol. 60, pp. 243-251, 2016.

[240] R. Steele, A. Lo, C. Secombe, and Y. K. Wong, "Elderly persons' perception and acceptance of using wireless sensor networks to assist healthcare," International journal of medical informatics, vol. 78, no. 12, pp. 788-801, 2009

[241] J. Meng, S. A. Hussain, D. C. Mohr, M. Czerwinski, and M. Zhang, "Exploring user needs for a mobile behavioral-sensing technology for depression management: qualitative study," Journal of medical Internet research, vol. 20, no. 7, p. e10139, 2018.

[242] M. Rabbi, M. Philyaw-Kotov, J. Lee, A. Mansour, L. Dent, X. Wang, R. Cunningham, E. Bonar, I. Nahum-Shani, P. Klasnja et al., "Sara: a mobile app to engage users in health data collection," in Proceedings of the 2017 ACM International Joint Conference on Pervasive and Ubiquitous Computing and Proceedings of the 2017 ACM International Symposium on Wearable Computers, 2017, pp. 781-789.

[243] Z. Fitz-Walter and D. Tjondronegoro, "Exploring the opportunities and challenges of using mobile sensing for gamification," in Proceedings of the UbiComp 11 Workshop on Mobile Sensing: Challenges, Opportunities and Future Directions 2011. ACM Press, 2011, pp. 1-5.

[244] H. E. Lee and J. Cho, "What motivates users to continue using diet and fitness apps? application of the uses and gratifications approach," Health communication, vol. 32, no. 12, pp. 1445-1453, 2017.

[245] D. N. Crowley, J. G. Breslin, P. Corcoran, and K. Young, "Gamification of citizen sensing through mobile social reporting," in 2012 IEEE International Games Innovation Conference. IEEE, 2012, pp. 1-5.

[246] A. L'Heureux, K. Grolinger, W. A. Higashino, and M. A. Capretz, "A gamification framework for sensor data analytics," in 2017 IEEE international congress on internet of things (ICIOT). IEEE, 2017, pp. 74-81.

[247] M. Floryan, P. I. Chow, S. M. Schueller, and L. M. Ritterband, "The model of gamification principles for digital health interventions: evaluation of validity and potential utility," Journal of medical Internet research, vol. 22, no. 6, p. e16506, 2020.

[248] C. Lee, K. Lee, and D. Lee, "Mobile healthcare applications and gamification for sustained health maintenance," Sustainability, vol. 9, no. 5, p. $772,2017$.

[249] K. Agrawal, M. Mehdi, M. Reichert, F. Hauck, W. Schlee, T. Probst, and R. Pryss, "Towards incentive management mechanisms in the context of crowdsensing technologies based on trackyourtinnitus insights," Procedia computer science, vol. 134, pp. 145-152, 2018.

[250] S. Kumar, W. J. Nilsen, A. Abernethy, A. Atienza, K. Patrick, M. Pavel, W. T. Riley, A. Shar, B. Spring, D. Spruijt-Metz et al., "Mobile health technology evaluation: the mhealth evidence workshop," American journal of preventive medicine, vol. 45, no. 2, pp. 228-236, 2013.

[251] S. Li, A. M. Psihogios, E. R. McKelvey, A. Ahmed, M. Rabbi, and S. Murphy, "Micro-randomized trials for promoting engagement in mobile health data collection: Adolescent/young adult oral chemotherapy adherence as an example," Current Opinion in Systems Biology, 2020.

[252] V. S. Dasari, B. Kantarci, M. Pouryazdan, L. Foschini, and M. Girolami, "Game theory in mobile crowdsensing: A comprehensive survey," Sensors, vol. 20, no. 7, p. 2055, 2020.

[253] D. Yang, G. Xue, X. Fang, and J. Tang, "Crowdsourcing to smartphones: Incentive mechanism design for mobile phone sensing," in Proceedings of the 18th annual international conference on Mobile computing and networking, 2012, pp. 173-184.

[254] G. Ji, Z. Yao, B. Zhang, and C. Li, "A reverse auction-based incentive mechanism for mobile crowdsensing," IEEE Internet of Things Journal, vol. 7, no. 9, pp. 8238-8248, 2020.

[255] H. Xiong, D. Zhang, L. Wang, and G. Chen, "Crowdrecruiter: Selecting participants for piggyback crowdsensing under probabilistic coverage constraint," in Proceedings of the 2014 ACM International Joint Conference on Pervasive and Ubiquitous Computing, 2014, pp. 703-714.

[256] H. Xiong, D. Zhang, G. Chen, L. Wang, and V. Gauthier, "Crowdtasker: Maximizing coverage quality in piggyback crowdsensing under budget constraint," in 2015 IEEE International Conference on Pervasive Computing and Communications (PerCom). IEEE, 2015, pp. 55-62.

[257] H. Xiong, D. Zhang, G. Chen, L. Wang, V. Gauthier, and L. E. Barnes, "icrowd: Near-optimal task allocation for piggyback crowdsensing," IEEE Transactions on Mobile Computing, vol. 15, no. 8, pp. 2010-2022, 2015.

[258] J. Wang, Y. Wang, D. Zhang, L. Wang, H. Xiong, A. Helal, Y. He, and F. Wang, "Fine-grained multitask allocation for participatory sensing with a shared budget," IEEE Internet of Things Journal, vol. 3, no. 6, pp. 1395-1405, 2016.

[259] J. Wang, Y. Wang, D. Zhang, F. Wang, H. Xiong, C. Chen, Q. Lv, and Z. Qiu, "Multi-task allocation in mobile crowd sensing with individual task quality assurance," IEEE Transactions on Mobile Computing, vol. 17, no. 9, pp. 2101-2113, 2018.

[260] J. Wang, J. Ma, Y. Wang, N. Wang, L. Wang, D. Zhang, F. Wang, and Q. Lv, "Will online digital footprints reveal your relationship status? an empirical study of social applications for sexual-minority men," Proceedings of the ACM on Interactive, Mobile, Wearable and Ubiquitous Technologies, vol. 4, no. 1, pp. 1-23, 2020.

[261] J. Dai, X. Bai, Z. Yang, Z. Shen, and D. Xuan, "Perfalld: A pervasive fall detection system using mobile phones," in 2010 8th IEEE International Conference on Pervasive Computing and Communications Workshops (PERCOM Workshops). IEEE, 2010, pp. 292-297. 
[262] M. Á. Á. de la Concepción, L. M. S. Morillo, J. A. Á. García, and L. González-Abril, "Mobile activity recognition and fall detection system for elderly people using ameva algorithm," Pervasive and Mobile Computing, vol. 34, pp. 3-13, 2017.

[263] A. A. N. Shirehjini, A. Yassine, and S. Shirmohammadi, "Equipment location in hospitals using rfid-based positioning system," IEEE Transactions on information technology in biomedicine, vol. 16, no. 6, pp. $1058-1069,2012$.

[264] A. C. Verceles and E. R. Hager, "Use of accelerometry to monitor physical activity in critically ill subjects: a systematic review," Respiratory care, vol. 60, no. 9, pp. 1330-1336, 2015.

[265] B. Fang, N. D. Lane, M. Zhang, A. Boran, and F. Kawsar, "Bodyscan: Enabling radio-based sensing on wearable devices for contactless activity and vital sign monitoring," in Proceedings of the 14th annual international conference on mobile systems, applications, and services, 2016, pp. 97-110.

[266] B. Fang, N. D. Lane, M. Zhang, and F. Kawsar, "Headscan: A wearable system for radio-based sensing of head and mouth-related activities," in 2016 15th ACM/IEEE International Conference on Information Processing in Sensor Networks (IPSN). IEEE, 2016, pp. 1-12.

[267] M. De Choudhury, M. Gamon, S. Counts, and E. Horvitz, "Predicting depression via social media," in Proceedings of the International AAAI Conference on Web and Social Media, vol. 7, no. 1, 2013.

[268] K. Saha, A. E. Bayraktaroglu, A. T. Campbell, N. V. Chawla, M. De Choudhury, S. K. D’Mello, A. K. Dey, G. Gao, J. M. Gregg, $\mathrm{K}$. Jagannath et al., "Social media as a passive sensor in longitudinal studies of human behavior and wellbeing," in Extended Abstracts of the 2019 CHI Conference on Human Factors in Computing Systems, 2019, pp. $1-8$.

[269] L. Canzian and M. Musolesi, "Trajectories of depression: unobtrusive monitoring of depressive states by means of smartphone mobility traces analysis," in Proceedings of the 2015 ACM international joint conference on pervasive and ubiquitous computing, 2015, pp. 1293-1304.

[270] Z. Wang, R. Guo, L. Hong, C. Wang, and L. Chen, "Demand-responsive windows scheduling in tertiary hospital leveraging spatiotemporal neural networks," in International Conference on Green, Pervasive, and Cloud Computing. Springer, 2020, pp. 231-243.

[271] M. De Choudhury, S. Counts, and E. Horvitz, "Social media as a measurement tool of depression in populations," in Proceedings of the 5th annual ACM web science conference, 2013, pp. 47-56.

[272] M. De Choudhury, E. Kiciman, M. Dredze, G. Coppersmith, and M. Kumar, "Discovering shifts to suicidal ideation from mental health content in social media," in Proceedings of the 2016 CHI conference on human factors in computing systems, 2016, pp. 2098-2110.

[273] A. Walton, I. Nahum-Shani, L. Crosby, P. Klasnja, and S. Murphy, "Optimizing digital integrated care via micro-randomized trials," Clinical Pharmacology \& Therapeutics, vol. 104, no. 1, pp. 53-58, 2018.

[274] N. J. Seewald, S. N. Smith, A. J. Lee, P. Klasnja, and S. A. Murphy, "Practical considerations for data collection and management in mobile health micro-randomized trials," Statistics in biosciences, vol. 11, no. 2 , pp. $355-370,2019$.

[275] F. Restuccia, N. Ghosh, S. Bhattacharjee, S. K. Das, and T. Melodia, "Quality of information in mobile crowdsensing: Survey and research challenges," ACM Transactions on Sensor Networks (TOSN), vol. 13, no. 4, pp. 1-43, 2017.

[276] Y. Li, J. Gao, C. Meng, Q. Li, L. Su, B. Zhao, W. Fan, and J. Han, "A survey on truth discovery," ACM Sigkdd Explorations Newsletter, vol. 17, no. 2, pp. 1-16, 2016.

[277] C. Meng, W. Jiang, Y. Li, J. Gao, L. Su, H. Ding, and Y. Cheng, "Truth discovery on crowd sensing of correlated entities," in Proceedings of the 13th acm conference on embedded networked sensor systems, 2015, pp. $169-182$.

[278] H. Mousa, S. B. Mokhtar, O. Hasan, O. Younes, M. Hadhoud, and L. Brunie, "Trust management and reputation systems in mobile participatory sensing applications: A survey," Computer Networks, vol. 90 , pp. 49-73, 2015.

[279] H. Jin, L. Su, D. Chen, K. Nahrstedt, and J. Xu, "Quality of information aware incentive mechanisms for mobile crowd sensing systems," in Proceedings of the 16th ACM International Symposium on Mobile Ad Hoc Networking and Computing, 2015, pp. 167-176.

[280] B. Guo, H. Chen, Z. Yu, W. Nan, X. Xie, D. Zhang, and X. Zhou, "Taskme: Toward a dynamic and quality-enhanced incentive mechanism for mobile crowd sensing," International Journal of Human-Computer Studies, vol. 102, pp. 14-26, 2017.

[281] N. Jiang, D. Xu, J. Zhou, H. Yan, T. Wan, and J. Zheng, "Toward optimal participant decisions with voting-based incentive model for crowd sensing," Information Sciences, vol. 512, pp. 1-17, 2020.
[282] M. Elf, M. Flink, M. Nilsson, M. Tistad, L. von Koch, and C. Ytterberg, "The case of value-based healthcare for people living with complex long-term conditions," BMC Health Services Research, vol. 17, no. 1, pp. 1-6, 2017.

[283] W. Wang, G. M. Harari, R. Wang, S. R. Müller, S. Mirjafari, K. Masaba, and A. T. Campbell, "Sensing behavioral change over time: Using within-person variability features from mobile sensing to predict personality traits," Proceedings of the ACM on Interactive, Mobile, Wearable and Ubiquitous Technologies, vol. 2, no. 3, pp. 1-21, 2018.

[284] B. Buck, K. A. Hallgren, E. Scherer, R. Brian, R. Wang, W. Wang, A. Campbell, T. Choudhury, M. Hauser, J. M. Kane et al., "Capturing behavioral indicators of persecutory ideation using mobile technology," Journal of psychiatric research, vol. 116, pp. 112-117, 2019.

[285] S. Jiménez-Serrano, S. Tortajada, and J. M. García-Gómez, "A mobile health application to predict postpartum depression based on machine learning," Telemedicine and e-Health, vol. 21, no. 7, pp. 567-574, 2015.

[286] G. Dong, M. Boukhechba, K. M. Shaffer, L. M. Ritterband, D. G. Gioeli, M. J. Reilley, T. M. Le, P. R. Kunk, T. W. Bauer, and P. I. Chow, "Using graph representation learning to predict salivary cortisol levels in pancreatic cancer patients," Journal of Healthcare Informatics Research, pp. 1-19, 2021

[287] P. T. Hertel and A. Mathews, "Cognitive bias modification: Past perspectives, current findings, and future applications," Perspectives on Psychological Science, vol. 6, no. 6, pp. 521-536, 2011.

[288] P. Delias, M. Doumpos, E. Grigoroudis, P. Manolitzas, and N. Matsatsinis, "Supporting healthcare management decisions via robust clustering of event logs," Knowledge-Based Systems, vol. 84, pp. 203-213, 2015.

[289] S. S. Intille, "Closing the evaluation gap in ubihealth research," IEEE pervasive computing, vol. 12, no. 2, pp. 76-79, 2013.

[290] C. I. Tang, I. Perez-Pozuelo, D. Spathis, and C. Mascolo, "Exploring contrastive learning in human activity recognition for healthcare," arXiv preprint arXiv:2011.11542, 2020.

[291] H. Sarker, M. Tyburski, M. M. Rahman, K. Hovsepian, M. Sharmin, D. H. Epstein, K. L. Preston, C. D. Furr-Holden, A. Milam, I. NahumShani et al., "Finding significant stress episodes in a discontinuous time series of rapidly varying mobile sensor data," in Proceedings of the 2016 CHI conference on human factors in computing systems, 2016, pp. 4489-4501.

[292] M. Shoaib, S. Bosch, O. D. Incel, H. Scholten, and P. J. Havinga, "Complex human activity recognition using smartphone and wrist-worn motion sensors," Sensors, vol. 16, no. 4, p. 426, 2016.

[293] H. M. Krumholz, "Big data and new knowledge in medicine: the thinking, training, and tools needed for a learning health system," Health Affairs, vol. 33, no. 7, pp. 1163-1170, 2014.

[294] J. F. Huckins, A. W. DaSilva, R. Wang, W. Wang, E. L. Hedlund, E. I. Murphy, R. B. Lopez, C. Rogers, P. E. Holtzheimer, W. M. Kelley et al., "Fusing mobile phone sensing and brain imaging to assess depression in college students," Frontiers in neuroscience, vol. 13, p. 248, 2019.

[295] T. J. Matarazzo, P. Santi, S. N. Pakzad, K. Carter, C. Ratti, B. Moaveni, C. Osgood, and N. Jacob, "Crowdsensing framework for monitoring bridge vibrations using moving smartphones," Proceedings of the IEEE, vol. 106 , no. 4, pp. 577-593, 2018.

[296] J. Schweitzer and C. Synowiec, "The economics of ehealth and mhealth," Journal of health communication, vol. 17, no. sup1, pp. 7381, 2012.

[297] L. W. Chang, J. Kagaayi, G. Nakigozi, D. Serwada, T. C. Quinn, R. H. Gray, R. C. Bollinger, S. J. Reynolds, and D. Holtgrave, "Cost analyses of peer health worker and mhealth support interventions for improving aids care in rakai, uganda," AIDS care, vol. 25, no. 5, pp. 652-656, 2013.

[298] M. Radha, P. Fonseca, A. Moreau, M. Ross, A. Cerny, P. Anderer, X. Long, and R. M. Aarts, "Sleep stage classification from heart-rate variability using long short-term memory neural networks," Scientific reports, vol. 9, no. 1, pp. 1-11, 2019.

[299] B. Guo, C. Chen, Z. Yu, D. Zhang, and X. Zhou, "Building humanmachine intelligence in mobile crowd sensing," It Professional, vol. 17, no. 3, pp. 46-52, 2015.

[300] S. Wan, Y. Zhao, T. Wang, Z. Gu, Q. H. Abbasi, and K.-K. R. Choo, "Multi-dimensional data indexing and range query processing via voronoi diagram for internet of things," Future Generation Computer Systems, vol. 91, pp. 382-391, 2019.

[301] H. G. Kayacik, M. Just, L. Baillie, D. Aspinall, and N. Micallef, "Data driven authentication: On the effectiveness of user behaviour modelling with mobile device sensors," arXiv preprint arXiv:1410.7743, 2014.

[302] M.-Y. Chih, T. Patton, F. M. McTavish, A. J. Isham, C. L. JudkinsFisher, A. K. Atwood, and D. H. Gustafson, "Predictive modeling of addiction lapses in a mobile health application," Journal of substance abuse treatment, vol. 46, no. 1, pp. 29-35, 2014 
[303] L. Ibraimi, M. Asim, and M. Petković, "Secure management of personal health records by applying attribute-based encryption," in Proceedings of the 6th International Workshop on Wearable, Micro, and Nano Technologies for Personalized Health. IEEE, 2009, pp. 71-74.

[304] W. Tang, J. Ren, K. Zhang, D. Zhang, Y. Zhang, and X. Shen, "Efficient and privacy-preserving fog-assisted health data sharing scheme," $A C M$ Transactions on Intelligent Systems and Technology (TIST), vol. 10, no. 6, pp. 1-23, 2019.

[305] A. K. Jain and D. Shanbhag, "Addressing security and privacy risks in mobile applications," IT Professional, vol. 14, no. 5, pp. 28-33, 2012.

[306] H. Zhu, H. Xiong, Y. Ge, and E. Chen, "Mobile app recommendations with security and privacy awareness," in Proceedings of the 20th ACM SIGKDD international conference on Knowledge discovery and data mining, 2014, pp. 951-960.

[307] R. Sandhu, E. Coyne, H. Feinstein, and C. Youman, "Role-based access control models," IEEE Computer, vol. 29, no. 2, pp. 38-47, 1996.

[308] A. P. Felt, E. Chin, S. Hanna, D. Song, and D. Wagner, "Android permissions demystified," in Proceedings of the 18th ACM conference on Computer and communications security, 2011, pp. 627-638.

[309] F. Roesner, T. Kohno, A. Moshchuk, B. Parno, H. J. Wang, and C. Cowan, "User-driven access control: Rethinking permission granting in modern operating systems," in 2012 IEEE Symposium on Security and Privacy, 2012, pp. 224-238.

[310] B. D. Mittelstadt, "Designing the health-related internet of things: Ethical principles and guidelines," Information-an International Interdisciplinary Journal, vol. 8, no. 3, p. 77, 2017.

[311] _ "Ethics of the health-related internet of things: a narrative review," Ethics and Information Technology, vol. 19, no. 3, pp. 157-175, 2017.

[312] D. Senesky, B. Jamshidi, K. B. Cheng, and A. Pisano, "Harsh environment silicon carbide sensors for health and performance monitoring of aerospace systems: A review," IEEE Sensors Journal, vol. 9, no. 11, pp. 1472-1478, 2009.

[313] S. M. R. Islam, D. Kwak, M. H. Kabir, M. Hossain, and K.-S. Kwak, "The internet of things for health care: A comprehensive survey," IEEE Access, vol. 3, pp. 678-708, 2015.

[314] M. A. G. Santos, R. Muñoz, R. Olivares, P. P. R. Filho, J. D. Ser, and V. H. C. de Albuquerque, "Online heart monitoring systems on the internet of health things environments: A survey, a reference model and an outlook," Information Fusion, vol. 53, pp. 222-239, 2020.

[315] H. Banaee, M. U. Ahmed, and A. Loutfi, "Data mining for wearable sensors in health monitoring systems: A review of recent trends and challenges," Sensors, vol. 13, no. 12, pp. 17472-17 500, 2013.

[316] W. Sun, Z. Cai, Y. Li, F. Liu, S. Fang, and G. Wang, "Security and privacy in the medical internet of things: A review," Security and Communication Networks, vol. 2018, pp. 1-9, 2018.

[317] B. Martínez-Pérez, I. De La Torre-Díez, and M. López-Coronado, "Mobile health applications for the most prevalent conditions by the world health organization: review and analysis," Journal of medical Internet research, vol. 15, no. 6, p. e120, 2013.

[318] H. Kalantarian, N. Alshurafa, and M. Sarrafzadeh, "A survey of diet monitoring technology," IEEE Pervasive Computing, vol. 16, no. 1, pp. 57-65, 2017. 